\title{
SCALE-INVARIANT GRAVITY: PARTICLE DYNAMICS
}

\author{
Julian Barbour \\ College Farm, South Newington, Banbury, Oxon, OX15 4JG, UK \\ Electronic address: julian@platonia.com
}

\begin{abstract}
A new and universal method for implementing scale invariance, called best matching, is presented. It extends to scaling the method introduced by Bertotti and the author to create a fully relational dynamics that satisfies Mach's principle. The method is illustrated here in the context of non-relativistic gravitational particle dynamics. It leads to far stronger predictions than general Newtonian dynamics. The energy and angular momentum of an 'island universe' must be exactly zero and its size, measured by its moment of inertia, cannot change. This constancy is enforced because the scale invariance requires all potentials to be homogeneous of degree -2. It is remarkable that one can nevertheless exactly recover the standard observed Newtonian laws and forces, which are merely accompanied by an extremely weak universal force like the one due to Einstein's cosmological constant. In contrast to Newtonian and Einsteinian dynamics, both the gravitational constant $\mathrm{G}$ and the strength of the cosmological force are uniquely determined by the matter distribution of the universe. Estimates of their values in agreement with observations are obtained. Best matching implements a dynamics of pure shape for which the action is a dimensionless number. If the universe obeys such scale-invariant law, steadily increasing inhomogeneity, not expansion of the universe, causes the Hubble red shift. The application of best matching to geometrodynamics is treated in a companion paper.
\end{abstract}




\section{Introduction}

The Euclidean symmetries of three-dimensional space (3-space) do not fully determine Newtonian kinematics. Absolute time and inertial frames are puzzling extra structures. They are eliminated in this paper in a dynamics that is fully invariant under Euclidean translations, rotations and dilatations. Besides results of independent interest, this paper introduces the techniques of the companion paper [1, which modifies Einstein's general relativity (GR), making it scale invariant in a similar manner. Both papers extend the 3-space approach of [2, 3], which derives spacetime kinematics, including the universal light cone and the gauge principle, from 3-space first principles.

Weyl [4] made the first serious attempt to create a scale-invariant (spacetime) dynamics, but his use of a conformally transformed 4-vector field to compensate conformal transformations of the gravitational field came to grief. Einstein [5] noted that it would make atoms emit spectral lines with history-dependent frequencies. This contradicted the observed facts. Many years later, Dirac [6] revived Weyl's idea in a simplified form in which the compensating 4-vector field is replaced by a simpler scalar compensating field. In his theory, there is no longer the flagrant disagreement with observation associated with Einstein's spectral argument. Nevertheless, Einstein would probably still have had reservations, since in Dirac's theory (which is a form of Brans-Dicke theory [7]) the coupling constants of the various forces of nature are epoch and location dependent. The strong equivalence principle (SEP), according to which the laws of nature, including the values of coupling constants, must be exactly the same everywhere and at every epoch, is violated. Dirac regarded this as a virtue, since he hoped it would justify his Large Numbers Hypothesis, but others would prefer the rigour of the SEP. From the late eighties, numerous authors have proposed Dirac-type theories [8]. The scalar compensating field is now widely called the dilaton, and it has been conjectured [8] that its vacuum expectation value determines the Planck length in an emergent fashion. A dilaton field is a necessary concomitant of the Einsteinian graviton in string theory, and there is now a vast literature on related matters.

There are reasons to be unhappy about the present situation. The general principles of physics impose few restrictions on scalar fields, so the scope for 'ad hocery' in order to match the latest observations is great. Even in string theory, the many different compactification possibilities lead to different dilatonic properties, and there seems to be little hope at this stage of unambiguous predictions for the form of the resulting effective four-dimensional theory. In addition, quantization, which often fails to respect classical scale invariance, introduces further uncertainty in the form of anomalies and arbitrary cutoffs.

In this and the companion paper [1, a new and universal approach to scale invariance is proposed. It makes no use of compensating fields at all and leads directly and naturally to a mechanism whereby the strong equivalence principle can be maintained. Since scalar fields play no essential role in the theory, there is much less scope for fudging. Scale-invariant gravity makes at least some unambiguous predictions, and it is therefore decidedly vulnerable to experimental disproof.

The idea behind scale-invariant gravity is in fact closely related to Weyl's original inspiration, which was to implement Descartes's dream of explaining all of physics by geometry. In 1919, despite Einstein's reservations, Weyl wrote [9]: "Descartes's dream of a purely geometrical physics is fulfilled in a remarkable way, though admittedly not one that he could have foreseen. In its concepts, physics in no way goes beyond geometry." However, years later he admitted defeat and wrote [10] "the facts of atomism teach us that length is not relative but absolute .. physics can never be reduced to geometry as Descartes had hoped." He came to this conclusion because the failure of his theory had persuaded him that physics did not respect the symmetries, like scaling, that one would expect it to inherit from space. He attributed the failure to the effect of quantum mechanics. As will be explained in this and the companion paper [1], scale-invariant gravity exploits 
the symmetries that so appealed to Weyl's intuition, but does so without compensating fields. Its realization in particle dynamics in this paper uses no concepts that, except for the Newtonian notion of point masses, go beyond those of the three-dimensional geometry axiomatized by Euclid. The geometrodynamical realization in [1] uses nothing but the concepts of modern three-dimensional differential geometry, above all Riemannian 3-spaces and scalar and 3-vector fields defined on them.

The key insight into scale-invariant gravity is the realization that the apparent breaking of scale invariance might not be due to quantum mechanics but to an inadequate theory of inertia. As currently described, inertia violates scale invariance. This fact seems to have escaped notice. Moreover, the defect can be remedied without compensating fields. Our approach to scale invariance is direct and, we believe, new. It is universal because it can be implemented for any spatial geometry that admits a similarity (scaling) symmetry.

The claim that the existing descriptions of inertia break scale invariance develops a criterion taken from Poincaré's discussion of absolute and relative motion in 11. It demands that, for any given spatial geometry, dynamics must be maximally predictive in a well-defined sense. Relationists like Mach [12] had argued that particle dynamics should be expressed solely using quantities invariant under Euclidean translations and rotations: the mutual separations $r_{i j}$ of the particles. But Poincaré pointed out that this can always be done through an automatic process of elimination. The real test is whether specification at an initial time of the $r_{i j}$ and their time derivatives $\dot{r}_{i j}$ (together with the particle masses and the force law) suffices to predict the future evolution (of an isolated system). Newtonian theory fails this precisely formulated prediction test because the $r_{i j}$ and $\dot{r}_{i j}$ contain no information about the total angular momentum $\mathbf{J}$, and very different evolutions result for different values of $\mathbf{J}$. As a result, when Newton's equations are expressed in terms of the $r_{i j}$ they contain third derivatives with respect to the time. Clearly, such a theory is less predictive than one containing only second derivatives. For more details, see [13].

Poincaré did not attempt to formulate a maximally predictive dynamics. Like Weyl later in the case of scaling, he simply accepted that nature did not respect mathematical intuition. This resignation may have been premature. Maximal predictive strength is a powerful constructive principle. $^{1}$ It imposes very characteristic structures on theories that possess the property. The papers 2, 3] have shown how it can explain some very basic known laws of nature: the universal light cone, the gauge principle, and Einstein's gravitational field equations. Now we use it in the hope of discovering new physics.

The first step is to push the principle to its limit. For Poincaré still allowed Newton's absolute time and length scale. Let us introduce configuration spaces. Let $\mathrm{T}$ be the one-dimensional space of Newtonian absolute times, and Q be the $3 N$-dimensional Newtonian configuration space of $N$ point particles of masses $m_{i}$. Let $\mathrm{Q}_{\mathrm{RCS}}$ (RCS stands for relative configuration space) be the $(3 N-6)$ dimensional space obtained from Q by quotienting with respect to the Euclidean translations and rotations. Thus, a point in $\mathrm{Q}_{\mathrm{RCS}}$ is the entire 6-dimensional group orbit in $\mathrm{Q}$ generated by them from a point $q \in Q$. All particle configurations that can be carried into each other by translations and rotations are identified. Finally, quotienting by the dilatations, we arrive at the $(3 N-7)$ dimensional space $\mathrm{Q}_{0}$ of shapes of $N$-particle configurations: shape space.

Standard Newtonian theory is formulated in the space $\mathrm{Q} \times \mathrm{T}$. Given the masses and the force law, its equations predict the future given an initial point $q t$ in $\mathrm{Q} \times \mathrm{T}$ and initial direction in $\mathrm{Q} \times \mathrm{T}$ at qt. (The direction in $\mathrm{Q}$ determines the direction of the momenta, and the slope in $\mathrm{T}$ their magnitude, i.e., the particle speeds.) Poincaré's criterion is met in $\mathrm{Q} \times \mathrm{T}$ but not in $\mathrm{Q}_{\mathrm{RCS}} \times \mathrm{T}$ and, a fortiori, not in $\mathrm{Q}_{0}$.

There are two known ways of constructing a maximally predictive particle dynamics. The first

\footnotetext{
${ }^{1}$ In Appendix II of [14] Einstein develops a notion of the 'strength' of dynamical theories that bears some relation to Poincaré's criterion. However, it is formulated in spacetime rather than configuration-space terms.
} 
uses directly the particle separations $r_{i j}$ or, in a scale-invariant theory, ratios of them. Schrödinger [15] was one of many who explored this route in the 20th century, but it leads to anisotropic effective masses that are ruled out by sensitive experiments [16. The absence of mass anisotropy is a very tough experimental test of relational theories. In [17, Bertotti and I proposed a maximally predictive form of variational dynamics that is appropriately called best matching. This method leads to isotropic masses and is presented in section 4. In particle dynamics, it leads to a small subset of the solutions allowed by Newton's equations. The conditions that select them show how elimination of non-relational kinematics leads to a dynamics that is fully determined by its underlying geometry. In [1], it leads to a scale-invariant generalization of GR: conformal gravity.

Each symmetry has its own distinctive best matching and imposes a constraint on the canonical momenta $\mathbf{p}_{i}$. Simultaneously, the best matching imposes further conditions on the Lagrangian and even on the solutions (in conformal gravity) that ensure propagation of the constraint. It is illuminating to exhibit the constraints and list the additional conditions.

Translational symmetry constrains the total momentum (all sums are over $i=1-N$ ):

$$
\mathbf{P}=\sum \mathbf{p}_{i}=0
$$

and the potential $U$ must be translational invariant, so Newton's third law is enforced. These are not new restrictions, since Galilean invariance enforces the invariance and ensures (11) holds in the centre-of-mass (c.m) frame.

Rotational best matching leads to the non-trivial vanishing of the total angular momentum $\mathbf{J}$ :

$$
\mathbf{J}=\sum \mathbf{x}_{i} \times \mathbf{p}_{i}=0
$$

and in conjunction with translational best matching enforces $U=U\left(r_{i j}\right)$. At this stage, the energy $E$ is arbitrary, and inertial motion, for which $U=$ constant, is possible. The results (11) and (2) were obtained in [17. The unique (vanishing) value of $\mathbf{J}$ imposed by best matching eliminates the ambiguity of prediction noted by Poincaré.

The extension of best matching to dilatations is new and leads to the most interesting result. It concerns the scalar quantity $D=\sum \mathbf{p}_{i} \cdot \mathbf{x}_{i}$, which has not, so far as I know, been given a name in the literature. Since it bears the same relation to expansion as angular momentum does to rotation (and has the same dimensions - action), I shall call it the dilatational momentum. Dilatational best matching forces it to vanish:

$$
D=\sum \mathbf{p}_{i} \cdot \mathbf{x}_{i}=0
$$

In addition, $U$ must be homogeneous of degree -2 in $r_{i j}$, and $E$ must be zero. Thus pure inertial motion, for which $U=$ constant and $E>0$, is not allowed. It is in this sense that inertia violates scaling. There is no maximally predictive inertial dynamics on shape space. One cannot formulate a theory of pure inertial motion without introducing additional kinematic structure - an absolute scale of length - that mathematical intuition suggests one should not employ. ${ }^{2}$ If one wishes to have any dynamics at all on shape space that satisfies the Poincaré criterion, it must include forces and have vanishing energy. This casts new light on the old problem highlighted by Hertz [18, 19]: why does energy exist in two disparate and independent forms, kinetic and potential? Their separate existence is the reason why pure inertial motion is allowed in Newtonian mechanics. In scale-invariant dynamics, this cannot be. The two forms of energy must come as inseparable twins and their sum must be zero. If the universe is scale invariant, this has implications for dimensional analysis and our understanding of the constants of nature (for a review of the present

\footnotetext{
${ }^{2}$ The situation in Newtonian mechanics is somewhat enigmatic. No absolute length itself can be observed (the overall scale is nominal), but the effect of change of length is observable. This matches what happens with regard to rotations. Newtonian mechanics is invariant under a time-independent rotation but not under changing rotations.
} 
understanding, see [20]). In fact, it leads to determination of the gravitational constant G (Secs. 6 and 7).

So far as I know, formulating a scale-invariant theory through a requirement on the form of the initial-value problem in shape space has not hitherto been considered. There is no indication of there having been work done in this direction in the often-cited review [21]. To avoid confusion, the reader should keep in mind this new criterion of scale invariance (or conformal invariance). It is used throughout both this paper and the companion [1].

Whereas $\mathbf{J}$, like $\mathbf{M}$, is conserved for isolated Newtonian systems, nothing forces $\mathbf{J}=0$. Moreover, the dilatational momentum $D$ is not even conserved in general let alone zero. The best-matching conditions are therefore non-trivial, and (3) is decidedly restrictive. Note also that (10)-(3) and the associated conditions on $U$ and $E$ are as purely geometrical in origin as the operators grad, curl and div of vector analysis, to which they exactly correspond. This reflects the universal geometrical nature of best matching.

It might seem that dilatational best matching is academic, since gravity and electrostatics have $1 / r$ potentials and not the $1 / r^{2}$ potentials required by (3). However, there exists a unique, natural and universal way to select $1 / r^{2}$ potentials that are manifested as effective $1 / r$ potentials indistinguishable from the Newtonian counterparts (section 5). The strong equivalence principle is then satisfied for all these forces, including gravity. The sole result of this procedure is to introduce a weak long-range force $\mathbf{F}$ like the one generated by Einstein's cosmological constant $\Lambda$. However, unlike $\Lambda$, the strength of $\mathbf{F}$ is uniquely determined by the potential of the system and is therefore epoch dependent. Moreover, $\mathbf{F}$ forces the 'size' of the universe, measured by the moment of inertia $I$ (and by the spatial volume $V$ in conformal gravity), to remain exactly constant. We shall see that there is a sense in which the cosmological force introduces an weak violation of the strong equivalence principle. It is, however, a violation that is reduced to the absolute minimum that is possible.

Another important thing to note is that the entire treatment of this and the companion paper [1] is based on the premise that the universe can be treated as a self-contained closed dynamical system. In the particle model, the moment of inertia of an $N$-particle system taken to represent an 'island universe' in Euclidean space plays an essential physical role in the theory. In conformal gravity [1, a similar role is played by the 3-volume of the universe. Scale invariance that respects the SEP relies on the use of ratios of local separations divided by global quantities: the moment of inertia and the 3-volume, respectively. Thus, the assumption of a self-contained universe is essential.

Section 2 shows how the condition (3) can arise as a very exceptional case within Newtonian theory and by how much Newtonian theory fails to be maximal predictive. Section 3 shows how time is eliminated by Jacobi's principle for the dynamical orbit of a system in its configuration space. (Dynamical orbits should not be confused with group orbits. Both exist in configuration space, and both play important roles in best matching.) Section 4 introduces best matching and develops the necessary formal techniques in Lagrangian form. Section 5 formulates the rule for passing from an arbitrary set of Newtonian potentials to scale-invariant counterparts with the associated cosmological force $\mathbf{F}$. Section 6 considers how dimensional analysis is changed by the elimination of time and an absolute scale of length. It also shows how scale invariance leads to an explicit expression for the gravitational constant G and the strength of F. Section 7 contains estimates of the strength of $\mathbf{F}$ predicted by scale-invariant gravity and shows how the explicit expression for $\mathrm{G}$ in terms of the matter distribution of the universe permits a determination of the mass and size of the universe from the empirical value of $\mathrm{G}$. Section 8 considers whether the actual universe is scale invariant. The greatest need is for an explanation of the Hubble red shift that does not rely on expansion of the universe. It is this that makes the theory vulnerable to experimental disproof. 
Section 9 considers possible quantum implications. Finally, the Appendix gives the Hamiltonian form of the theory.

\section{The Lagrange-Jacobi Relation}

In the c.m frame, let an isolated system have Lagrangian $\mathcal{L}=T-U$ with $T=\sum\left(m_{i} / 2\right) \dot{\mathbf{x}}_{i} \cdot \dot{\mathbf{x}}_{i}$ and potential $U$. The energy $E=T+U$ is conserved. The c.m moment of inertia $I$ is

$$
I=\sum m_{i} \mathbf{x}_{i} \cdot \mathbf{x}_{i} \equiv \frac{1}{M} \sum_{i<j} m_{i} m_{j} r_{i j}^{2}, \quad M=\sum m_{i}
$$

Lagrange and Jacobi noted ${ }^{3}$ that $\ddot{I}=2 \sum m_{i} \dot{\mathbf{x}}_{i} \cdot \dot{\mathbf{x}}_{i}+2 \sum m_{i} \mathbf{x}_{i} \cdot \ddot{\mathbf{x}}_{i}$ has important properties. By Newton's second law $m_{i} \ddot{\mathbf{x}}_{i}=-\partial U / \partial \mathbf{x}_{i}$ and the definition of $T$, we find $\ddot{I}=4 T-2 \sum \mathbf{x}_{i} \cdot \frac{\partial U}{\partial \mathbf{x}_{i}}$. If now $U$ is homogeneous of degree $k$, then, using Euler's theorem and $T=E-U$, we find

$$
\ddot{I}=4(E-U)-2 k U \text {. }
$$

Consider Newtonian celestial mechanics, for which $k=-1$. Then $\ddot{I}=4 E-2 U$, from which Lagrange deduced the first qualitative result in dynamics. Since $U<0$ for gravity, $E \geq 0$ implies $\ddot{I}>0$. Thus $I$ is concave upwards and must tend to infinity as $t \longrightarrow+\infty$ and $t \longrightarrow-\infty$. In turn, this means that at least one of the interparticle distances must increase unboundedly, so that any system with $E \geq 0$ is unstable.

Another consequence of (51) is the virial theorem. For suppose that the system has virialized, so that $I \approx 0$. Then $4 E=(2 k+4) U$.

For our purposes, the most interesting consequence of (15) arises when $k=-2$. For then

$$
\ddot{I}=4 E \text {. }
$$

Thus, $I$ has the parabolic dependence $I=2 E t^{2}+b t+c$ on the time and will tend rapidly to zero or infinity. Such a system is extremely unstable, either imploding or exploding.

However, suppose $E=0$. Then $\ddot{I}=0$ by (6) , so that $\dot{I}=2 \sum m_{i} \dot{\mathbf{x}}_{i} \cdot \mathbf{x}_{i}=2 \sum \mathbf{p}_{i} \cdot \mathbf{x}_{i}=$ constant. Thus, the dilatational momentum $D=\sum \mathbf{p}_{i} \cdot \mathbf{x}_{i}$ is conserved if both $U$ is homogeneous of degree -2 and $E=0$. (The arbitrary additive constant in $E$ is fixed by requiring $U \longrightarrow 0$ when all $r_{i j} \longrightarrow 0$.) Obtained thus, conservation of $D$ is a fluke. However, as we just noted, dilatational best matching requires not merely conservation but vanishing of $D$. The homogeneity of $U$ and vanishing of $E$ are enforced by the symmetry. Homogeneity is crucial for scale invariance.

Newtonian dynamics has always been seen as the paradigm of rationality. However, Poincaré's analysis [1] of the initial-value problem shows that rationality is 'arena dependent'. If one treats the universe as an isolated dynamical system in shape space, Newtonian dynamics needs more initial data than seem necessary. It is worth spelling this out for the three-body problem, for which shape space $\mathrm{Q}_{0}$ is the two-dimensional space of triangle shapes. Let it be coordinatized by two angles $\alpha$ and $\beta$ of the triangle. If they are taken as dependent and independent variables, respectively, the orbit in $\mathrm{Q}_{0}$ for a theory that satisfies the Poincaré criterion should be described by

$$
\frac{\mathrm{d}^{2} \alpha}{\mathrm{d} \beta^{2}}=f\left(\alpha, \beta, \frac{\mathrm{d} \alpha}{\mathrm{d} \beta}\right)
$$

and three initial data would be needed: $\alpha, \beta, \mathrm{d} \alpha / \mathrm{d} \beta$.

\footnotetext{
${ }^{3}$ I understand from $N$-body specialists $[22]$ that the relation (5) of this section is generally known as the LagrangeJacobi relation. For a useful account of Lagrange's work, see 23]
} 
Newtonian theory fails this ideal of a dynamics of pure shape. To determine the curve of a generic solution in $\mathrm{Q} \times \mathrm{T}$ projected down to $\mathrm{Q}_{0}$, five further data are needed. Two fix the direction of $\mathbf{J}$ relative to the instantaneous triangle, one is the constant ratio (rotational K.E/total K.E), another is the varying ratio $T / U$, and the final one is the varying ratio (K.E in change of size/K.E in change of shape). There is a similar mismatch for the $N$-body problem. All the extra data look natural in the Newtonian arena but incongruous in shape space. Rotational best matching eliminates the first three, dilatational best matching the other two.

\section{Jacobi's Principle}

The first step to a dynamics of pure shape is the elimination of time by Jacobi's principle [19], which describes all Newtonian motions of one $E$ as geodesics on configuration space. This topic has already been discussed in [2, 24, 25], so the ground already covered there will be only briefly recapitulated. However, this section will also consider the important issue of dimensions in a timeless and scale-invariant theory, which has not hitherto been discussed.

For $N$ particles of masses $m_{i}$ with potential $U\left(\mathbf{x}_{1}, \ldots, \mathbf{x}_{N}\right)$ and energy $E$, the Jacobi action is [19]

$$
I_{\mathrm{Jacobi}}=2 \int \sqrt{E-U} \sqrt{\tilde{T}} \mathrm{~d} \lambda
$$

where $\lambda$ labels the points on trial curves and $\tilde{T}=\sum \frac{m_{i}}{2} \frac{\mathrm{d} \mathbf{x}_{i}}{\mathrm{~d} \lambda} \cdot \frac{\mathrm{d} \mathbf{x}_{i}}{\mathrm{~d} \lambda}$ is the parametrized kinetic energy. The action (8) is timeless since the label $\lambda$ could be omitted and the mere displacements $d \mathbf{x}_{i}$ employed, as is reflected in the invariance of $I_{\mathrm{Jacobi}}$ under the reparametrization

$$
\lambda \rightarrow f(\lambda) .
$$

In fact, it is much more illuminating to write the Jacobi action in the form

$$
I_{\mathrm{Jacobi}}=2 \int \sqrt{E-U} \sqrt{T^{*}}, \quad T^{*}=\sum \frac{m_{i}}{2} \mathrm{~d} \mathbf{x}_{i} \cdot \mathrm{d} \mathbf{x}_{i},
$$

which makes its timeless nature obvious and dispenses with the label $\lambda$.

The characteristic square roots of $I_{\mathrm{Jacobi}}$ fix the structure of the canonical momenta:

$$
\mathbf{p}_{i}=\frac{\partial \mathcal{L}}{\partial\left(\mathrm{d} \mathbf{x}_{i} / \mathrm{d} \lambda\right)}=m_{i} \sqrt{\frac{E-U}{\tilde{T}}} \frac{d \mathbf{x}_{i}}{d \lambda},
$$

which, being homogeneous of degree zero in the velocities, satisfy the constraint 26.

$$
\sum \frac{\mathbf{p}_{i} \cdot \mathbf{p}_{i}}{2 m_{i}}=E-U
$$

The Euler-Lagrange equations are

$$
\frac{\mathrm{d} \mathbf{p}^{i}}{\mathrm{~d} \lambda}=\frac{\partial \mathcal{L}}{\partial \mathbf{x}_{i}}=-\sqrt{\frac{\tilde{T}}{E-U}} \frac{\partial U}{\partial \mathbf{x}_{i}},
$$

where $\lambda$ is still arbitrary. If we choose it such that

$$
\frac{\tilde{T}}{E-U}=1 \Rightarrow \tilde{T}=E-U
$$


then (111) and (13) become

$$
\mathbf{p}_{i}=m_{i} \frac{\mathrm{d} \mathbf{x}_{i}}{\mathrm{~d} \lambda}, \quad \frac{\mathrm{d} \mathbf{p}_{i}}{\mathrm{~d} \lambda}=-\frac{\partial U}{\partial \mathbf{x}_{i}},
$$

and we recover Newton's second law w.r.t this special $\lambda$. However, (14), which is usually taken to express energy conservation, becomes the definition of time. Indeed, this emergent time, chosen to make the equations of motion take their simplest form [27, is the astronomers' operational ephemeris time [28]. It is helpful to see how 'change creates time'. The increment $\delta t$ generated by displacements $\delta \mathbf{x}_{i}$ is

$$
\delta t=\frac{\sqrt{\sum m_{i} \delta \mathbf{x}_{i} \cdot \delta \mathbf{x}_{i}}}{\sqrt{2(E-U)}} \equiv \frac{\delta s}{\sqrt{2(E-U)}} .
$$

Each particle 'advances time' in proportion to the square root of its mass and to its displacement, the total contribution $\delta s$ being weighted by $\sqrt{2(E-U)}$. In a scale-invariant theory, $E=0$, and $\delta t$ takes an especially suggestive form: a given kinetic $\delta s$ 'advances time' by an amount inversely proportional to $|U|$.

\section{Best Matching}

Our goal is a theory of Jacobi-type geodesics on shape space $\mathrm{Q}_{0}$. To do this, we might interpolate shapes $A_{0}, B_{0} \in \mathrm{Q}_{0}$ with suitably continuous curves $q_{0}(\lambda)$ and extremalize an action of the form

$$
I_{\mathrm{Q}_{0}}=\int_{\lambda_{A_{0}}}^{\lambda_{B_{0}}} \mathrm{~d} \lambda \sqrt{f_{i j} \frac{\mathrm{d} q_{0}^{i}}{\mathrm{~d} \lambda} \frac{\mathrm{d} q_{0}^{j}}{\mathrm{~d} \lambda}}
$$

w.r.t certain shape coordinates $q_{0}^{i}, \ldots, q_{0}^{3 N-7}$ on $\mathrm{Q}_{0}$. The $f_{i j}$ will be functions of the shape coordinates, $f_{i j}=f_{i j}\left(q_{0}^{1}, \ldots, q_{0}^{3 N-7}\right)$, and the usual summation is understood. But shape coordinates are awkward, and this approach is impracticable.

Next, we try to work in $\mathrm{Q}$ and attempt an action of similar form:

$$
I_{\mathrm{Q}}=\int_{\lambda_{A}}^{\lambda_{B}} \mathrm{~d} \lambda \mathcal{L}, \quad \mathcal{L}=\sqrt{f_{i j} \frac{\mathrm{d} q^{i}}{\mathrm{~d} \lambda} \frac{\mathrm{d} q^{j}}{\mathrm{~d} \lambda}},
$$

where now $i=1, \ldots, 3 N$ and $f_{i j}=f_{i j}(q)$. Since $I_{\mathrm{Q}}$ should depend only on the projected curve $q_{0}(\lambda)$ in $\mathrm{Q}_{0}$, we seek to make $\mathcal{L}$ invariant under $\lambda$-dependent transformations that shift the $q(\lambda)$ 's by arbitrary $\lambda$-dependent amounts along their group orbits. The $\lambda$-dependence is decisive.

Let us consider translations in one dimension and infinitesimally differing $A$ and $B$. Then $\lambda_{B}-\lambda_{A}=\delta \lambda$ can be infinitesimal, and to first order we shall be minimizing

$$
I_{\mathrm{TS}}=\sqrt{f_{i j} \delta q^{i} \delta q^{j}}, \quad \delta q^{i}=\frac{\mathrm{d} q^{i}}{\mathrm{~d} \lambda} \delta \lambda,
$$

where TS stands for thin sandwich. The action (16) must be stationary to first order under

$$
q^{i}(\lambda) \longrightarrow q^{i}(\lambda)+b(\lambda) e^{i}
$$

for all choices of $b(\lambda)$. Here $e^{i}=1$ for all $i$ is the generator of translations for particle $i$. If this is so, $I_{\text {TS }}$ will depend only on the orbits of $A$ and $B$, since different choices of $b(\lambda)$ move $A$ and $B$ arbitrarily along their orbits. Note that under (17) the velocities change:

$$
v^{i} \longrightarrow v^{i}+b^{\prime} e^{i}, \quad v^{i}=\frac{\mathrm{d} q^{i}}{\mathrm{~d} \lambda}, \quad b^{\prime}=\frac{\mathrm{d} b}{\mathrm{~d} \lambda} .
$$


Let us make a Taylor expansion of $b(\lambda)$ :

$$
b(\lambda)=b(0)+b^{\prime}(0) \delta \lambda+\ldots
$$

The thin-sandwich problem (TSP) requires us to minimize (16) to first order w.r.t $b(0)$ and $b^{\prime}(0)$. The $\lambda$-dependence of the transformations is reflected in the fact that for the TSP these are to be treated as independent Lagrange multipliers. The first shifts $A$ and $B$ together along their orbits; the second displaces one relative to the other. This twofold freedom gives rise to a universal characteristic structure of such variational problems. Provided the TSP has a unique solution, ${ }^{4}$ its limit as $\delta \lambda \longrightarrow 0$ will clearly define a metric on Q. For it can be used to define the 'distance' $I_{\text {TS }}$ (16) between any two neighbouring points in $Q_{0}$. This process gets its name because the 'distance' is obtained when the configurations are best matched: brought as near to congruence, as measured by (16), as possible.

To implement best matching in a convenient formal scheme, we introduce an auxiliary gauge variable $a \in \Gamma$, where $\Gamma$ for one-dimensional translations is $R^{1}$. However, for a general symmetry group defined by $d$ parameters, the auxiliary space $\Gamma$ will have $d$ dimensions $(d=7$ for the full Euclidean group). The space $\Gamma$ is used to extend the configuration space $Q$ to a larger formal space $\mathrm{Q} \times \Gamma .{ }^{5}$ Since $\mathrm{Q}$ contains $d$ unphysical degrees of freedom, $d$ is the dimension of the group orbits that foliate $\mathrm{Q}$ into $\mathrm{Q}_{0}$ and is the degeneracy. Thus, the extension doubles the degeneracy. We shall denote the coordinates on $\Gamma$ collectively by $a$, which is a single variable in the present case.

We now use the auxiliary a to define the corrected coordinate 6

$$
\bar{q}^{i}=q^{i}+a e^{i}
$$

It is invariant under the pair of mutually compensating transformations:

$$
q^{i}(\lambda) \longrightarrow q^{i}(\lambda)+b(\lambda) e^{i}, \quad a(\lambda) \longrightarrow a(\lambda)-b(\lambda)
$$

which, in view of the triviality of the invariance thereby achieved, we shall call the banal transformation. It induces equally trivial mutually compensating transformations of the velocities:

$$
v^{i} \longrightarrow v^{i}+b^{\prime} e^{i}, \quad a^{\prime} \longrightarrow a^{\prime}-b^{\prime}, \quad a^{\prime}=\frac{\mathrm{d} a}{\mathrm{~d} \lambda} .
$$

We now define the corrected velocity $\bar{v}^{i}$ :

$$
\bar{v}^{i}=v^{i}+a^{\prime} e^{i}
$$

Unlike the naive (uncorrected) coordinate $q^{i}$ and the naive velocity $v^{i}$, both $\bar{q}^{i}$ and $\bar{v}^{i}$ are invariant under the banal transformation (20). One correction compensates the other. It is important to distinguish the transformation parameter $b$ from the auxiliary $a$. Although they seem to 'do the same thing' to the $q^{i}$ 's [cf. (17) and (19)], their conceptual status is quite different.

Two rules now yield a fully invariant action of curves on $\mathrm{Q}_{0}: 1$ ) On $\mathrm{Q} \times \Gamma$, choose a Lagrangian $\overline{\mathcal{L}}=\overline{\mathcal{L}}\left[\bar{q}^{i}, \bar{v}^{i}\right]$ that is the square root of a functional homogeneously quadratic in the $\bar{v}^{i}$ and otherwise depends only on the $\bar{q}^{i} ; 2$ ) ensure that the action along any curve in $\mathrm{Q} \times \Gamma$ is invariant w.r.t arbitrary variations $\delta a$ of $a$. The 'arbitrary' means that there are no fixed end points of the $a$ variation. The

\footnotetext{
${ }^{4}$ It will in the particle model, since positive-definite quantities are being minimized; the situation remains open in scale-invariant geometrodynamics [1], which however has a well-behaved Hamiltonian formulation.

${ }^{5}$ The auxiliary space $\Gamma$ is a fibre over the base Q, but the formalism of fibre bundles is not needed for translations and dilatations. It is for rotations, which are only mentioned in this paper. For a beautiful gauge fibre-bundle treatment of rotations in the $N$-body problem, see [29].

${ }^{6}$ We shall use the words coordinate, velocity, and momentum in the sense of Hamiltonian canonical theory.
} 
point is that the $a$ variation is effectively being used to solve successive TSPs to determine the metric on $Q_{0}$. Were we to fix $a$ at the end points, we could not determine the metric at them. We shall say that $a$ is varied by the free-end-point method. The $q$ variation is different. It is being used to find the geodesics w.r.t the metric determined by the $a$ variation.

Free-end-point variation has consequences of three kinds: 1) Conditions on the $\bar{q}$ dependence of $\overline{\mathcal{L}} ; 2)$ constraints on the canonical momenta that the Euler-Lagrange equations propagate because $\overline{\mathcal{L}}$ satisfies conditions 1); 3) subsidiary equations that must be satisfied in addition to the constraints and the Euler-Lagrange equations. (This happens in GR and conformal gravity [1.)

The conditions on the $\bar{q}$ dependence of $\overline{\mathcal{L}}$ imposed under 1) can often cause the auxiliary $a$ to disappear from $\overline{\mathcal{L}}$ (its velocity $a^{\prime}$ never does). This happens for translations, as we shall soon see, and rotations and in Abelian and non-Abelian gauge theories. ${ }^{7}$ However, $a$ appears explicitly in scale-invariant theories and also in a representation of GR [1].

The reason for this is a characteristic property of scaling transformations, which, in contrast to the additive nature of the translations (17), are multiplicative and change the norm. Indeed, the compensating transformations for dilatations are

$$
q^{i} \longrightarrow b q^{i}, \quad a \longrightarrow \frac{a}{b}
$$

The corrected coordinate and corrected velocity are

$$
\bar{q}^{i}=a q^{i}, \quad \bar{v}^{i}=a v^{i}+q^{i} a^{\prime} .
$$

The device of replacing the 'partly redundant' $q^{i}$ by corrected $\bar{q}^{i}$ is evidently universally applicable, i.e., it is always possible to introduce a compensating transformation like the second members of (20) and (23) (which are both 'inverses' - one by subtraction, the other by division). More complicated symmetries and several simultaneous symmetries are beyond the scope of this paper. However, translations and dilatations in particle mechanics are a useful and adequate preparation for the conformal symmetry in [1]. For simplicity, we take the Lagrangian $\overline{\mathcal{L}}\left[\bar{q}^{i}, \bar{v}^{i}\right]$ diagonal in the $\bar{v}^{i}:$

$$
I_{\mathrm{BM}}=\int \mathrm{d} \lambda \overline{\mathcal{L}}\left[\bar{q}^{i}, \bar{v}^{i}\right]=\int \mathrm{d} \lambda 2 \sqrt{B\left(\bar{q}^{1}, \ldots, \bar{q}^{N}\right) T}, \quad T=\frac{1}{2} \sum_{i=1}^{N} m_{i}\left(\bar{v}^{i}\right)^{2} .
$$

While we shall always insist on free-end-point variation w.r.t $a$, the remaining Euler-Lagrange part of the variation can be performed in two different ways. We can vary either w.r.t the $q^{i}$ or w.r.t the $\bar{q}^{i}$. The latter variation is neater, but we still use the former, since it shows how the absolute part of Newtonian kinematics, i.e., the part not dictated by three-dimensional Euclidean geometry, arises.

The physical variables $q^{i}$ and the auxiliary variable $a$ have the canonical momenta

$$
p^{i} \equiv \frac{\partial \overline{\mathcal{L}}}{\partial v^{i}}=\sqrt{\frac{B}{T}} \frac{\partial T}{\partial \bar{v}^{i}} \frac{\partial \bar{v}^{i}}{\partial v^{i}}, \quad p^{a} \equiv \frac{\partial \overline{\mathcal{L}}}{\partial a^{\prime}}=\sum_{i} \sqrt{\frac{B}{T}} \frac{\partial T}{\partial \bar{v}^{i}} \frac{\partial \bar{v}^{i}}{\partial a^{\prime}} .
$$

Substituting the values of $\partial \bar{v}^{i} / \partial v^{i}$ and $\partial \bar{v}^{i} / \partial a^{\prime}$ for translations and dilatations, we find

$$
p_{\mathrm{t}}^{i}=\sqrt{\frac{B}{T}} \frac{\partial T}{\partial \bar{v}^{i}}, \quad p_{\mathrm{t}}^{a}=\sum_{i} \sqrt{\frac{B}{T}} \frac{\partial T}{\partial \bar{v}^{i}} ; \quad p_{\mathrm{d}}^{i}=\sqrt{\frac{B}{T}} \frac{\partial T}{\partial \bar{v}^{i}} a, \quad p_{\mathrm{d}}^{a}=\sum_{i} \sqrt{\frac{B}{T}} \frac{\partial T}{\partial \bar{v}^{i}} q^{i} .
$$

\footnotetext{
${ }^{7} \mathrm{~A}$ full treatment of this point goes beyond the scope of this paper. I hope to discuss it in a further paper devoted to the unifying perspective on gauge theory that best matching provides.
} 
Therefore, in the respective cases we have the constraints

$$
\sum_{i} p_{\mathrm{t}}^{i} \equiv P_{\mathrm{t}}=p_{\mathrm{t}}^{a}, \quad \sum_{i} p_{\mathrm{d}}^{i} q^{i} \equiv D=a p_{\mathrm{d}}^{a},
$$

which are primary [26] because they arise from the mere form of the action and not through variation (which gives rise to secondary constraints). The constraints (27) reflect the banal invariance of the action. They are homogeneously linear in the $p^{i}$ with coefficients solely determined by the symmetry and arise because $\overline{\mathcal{L}}$ depends on the corrected velocities $\bar{v}^{i}$. The actual form of $\overline{\mathcal{L}}$ is immaterial.

In accordance with the free-end-point method, the action must be stationary with respect to arbitrary variations of $a$. This means that at any instant both $\partial \overline{\mathcal{L}} / \partial a=0$ and $\partial \overline{\mathcal{L}} / \partial a^{\prime}=0$. But $\partial \overline{\mathcal{L}} / \partial a^{\prime}$ is the canonical momentum $p^{a}$ of the auxiliary variable, which must therefore vanish as a secondary constraint. The primary constraints (27) now become

$$
P_{\mathrm{t}}=0, \quad D=0
$$

i.e., the momentum and dilatational momentum must vanish, as promised at the end of the Introduction.

As Dirac points out [26, as soon as one has constraints in any dynamical system, one must verify that they are propagated by the Euler-Lagrange equations. An important feature of best matching is the manner in which its first condition $\partial \overline{\mathcal{L}} / \partial a=0$ automatically ensures propagation of the constraint enforced by its second condition $\partial \overline{\mathcal{L}} / \partial a^{\prime}=0$. We can see this in two ways.

First, since the action must be stationary for all variations of $a$, they will include variations with fixed end points, from which we deduce the standard Euler-Lagrange equation

$$
\frac{\mathrm{d} p^{a}}{\mathrm{~d} \lambda}=\frac{\partial \overline{\mathcal{L}}}{\partial a}
$$

Since we also require $\partial \overline{\mathcal{L}} / \partial a=0$, this ensures that $p^{a}$ will be conserved. Thus, if $p^{a}$ is initially zero, it will remain zero. By the primary constraints (27), the relations that hold for $p^{a}$ must also hold for the corresponding combinations $P_{\mathrm{t}}$ and $D$ of the physical canonical momenta, so the constraints (28) must propagate.

Second, we can, of course, confirm the propagation of (28) directly, using the Euler-Lagrange equations of the physical variables in conjunction with the condition $\partial \overline{\mathcal{L}} / \partial a=0$. It is worth doing this to see the explicit form of this condition. In the case of translations

$$
\frac{\partial \overline{\mathcal{L}}}{\partial a}=\sum_{i} \frac{\partial \overline{\mathcal{L}}}{\partial \bar{q}^{i}} \frac{\partial \bar{q}^{i}}{\partial a}=\sum_{i} \sqrt{\frac{B}{T}} \frac{\partial B}{\partial \bar{q}^{i}}=0 .
$$

From the Euler-Lagrange equations

$$
\frac{\mathrm{d} p^{i}}{\mathrm{~d} \lambda}=\sqrt{\frac{B}{T}} \frac{\partial B}{\partial \bar{q}^{i}},
$$

we directly conclude that

$$
\frac{\mathrm{d} P_{\mathrm{t}}}{\mathrm{d} \lambda}=\sum_{i} \frac{\mathrm{d} p^{i}}{\mathrm{~d} \lambda}=\sum_{i} \sqrt{\frac{B}{T}} \frac{\partial B}{\partial \bar{q}^{i}},
$$

which does indeed vanish by virtue of (29). Of course, (29) is the condition for the potential $B$ to be translationally invariant (which ensures fulfilment of Newton's third law). One of the easiest ways to achieve this is to choose

$$
B=B\left(\left|\bar{q}^{i}-\bar{q}^{j}\right|\right)=B\left(\left|q^{i}-q^{j}\right|\right),
$$


the second equation holding because the correcting $a e^{i}$ in the $\bar{q}^{i}$ simply drops out of the difference. Thus, for corrected coordinates formed additively the potential will not depend on the auxiliary gauge variable $a$. Also any constant $E$ can be added to the potential $B$, matching the total energy that appears in the Jacobi action (8).

In the case of dilatations, we have a much more intimate interconnection of conditions. The condition $\partial \overline{\mathcal{L}} / \partial a=0$ gives

$$
0=\frac{\partial \overline{\mathcal{L}}}{\partial a} \equiv \sum_{i}\left(\sqrt{\frac{T}{B}} \frac{\partial B}{\partial \bar{q}^{i}} \frac{\partial \bar{q}^{i}}{\partial a}+\sqrt{\frac{B}{T}} \frac{\partial T}{\partial \bar{v}^{i}} \frac{\partial \bar{v}^{i}}{\partial a}\right)=\sum_{i}\left(\sqrt{\frac{T}{B}} \frac{\partial B}{\partial \bar{q}^{i}} q^{i}+\sqrt{\frac{B}{T}} \frac{\partial T}{\partial \bar{v}^{i}} v^{i}\right) .
$$

We now divide by $a$ and add and subtract $D a^{\prime} / a$, where $D$ is the dilatational constraint (dilatational momentum):

$$
0=\frac{1}{a} \sum_{i}\left(\sqrt{\frac{T}{B}} \frac{\partial B}{\partial \bar{q}^{i}} a q^{i}+\sqrt{\frac{B}{T}} \frac{\partial T}{\partial \bar{v}^{i}}\left(a v^{i}+q^{i} a^{\prime}\right)\right)-\frac{1}{a} \sum_{i} \sqrt{\frac{B}{T}} \frac{\partial T}{\partial \bar{v}^{i}} q^{i} a^{\prime} .
$$

Since $a v^{i}+q^{i} a^{\prime}=\bar{v}^{i},\left(\partial T / \partial \bar{v}^{i}\right) \bar{v}^{i}=2 T$ and the constraint is required to vanish, we conclude

$$
\sum_{i}\left(\sqrt{\frac{T}{B}} \frac{\partial B}{\partial \bar{q}^{i}} \bar{q}^{i}+\sqrt{\frac{B}{T}} 2 T\right)=0 \Rightarrow \sum_{i}\left(\frac{\partial B}{\partial \bar{q}^{i}} \bar{q}^{i}+2 B\right)=0 .
$$

By Euler's theorem, this tells us that the scheme will be consistent if $B$ is homogeneous of degree -2 in the $\bar{q}^{i}$ 's. The last step in establishing the mutual consistency of our interlocking system of equations is to note that the propagation of the dilatational constraint by the Euler-Lagrange equations leads to exactly the same condition (30).

To make the final connection with the special Newtonian situation summarized at the end of section 1 and derived from the Lagrange-Jacobi relation in section 2, we now exploit the freedom to make a banal transformation and so pass from a general frame of reference to the unique distinguished representation in which $a=1$ and $\lambda$ is chosen to make $B / T=1$. In the distinguished representation, we recover Newton's laws exactly subject to the homogeneity condition on the potential and the dilatational constraint that the dilatational momentum vanishes. The $q^{i}$ are then coordinates in an inertial frame of reference, and the special $\lambda$ is indistinguishable from Newton's absolute time. We have derived the non-geometrical (absolute) part of Newtonian kinematics from a purely relational scheme.

As mentioned in the introduction, a similar treatment for the rotation group [17, 29] yields the constraint that the total c.m angular momentum vanishes (and a further restriction on the potential, which must be a function of the inter-particle separations). Note how well the relational origin of this dynamics is hidden. The constraints and the condition $E=0$ apply to the complete 'island universe' of $N$ particles in Euclidean space, and need not apply to subsystems like the solar system - only the sums over all subsystems need satisfy the constraints. Since it is difficult to verify the constraints and the condition $E=0$ for the complete universe, the sole hard evidence for the relational origin of the dynamics is in the form of the observed potentials. The conditions imposed by the translations and rotations are respected by the observed Newtonian approximations to relativistic physics, but there seems to be a spectacular failure to match the condition needed for a fully scale-invariant dynamics. The next section will show how it may be only an apparent failure. However, let me first conclude this section with two comments.

The first concerns the somewhat elaborate formalism of the free-end-point method. Why is it necessary? The velocity of our auxiliary variable plays exactly the same role as the scalar potential 
$\phi$ in the Hamiltonian formulation of Maxwell theory. Since $\phi$ has the dimensions of velocity and the action contains no variable of which it is the velocity, it should strictly be treated as an ignorable coordinate [19. It is, however, habitually treated as a multiplier, variation with respect to which yields the Gauss constraint, which is a stronger result than would follow from regarding $\phi$ as an ignorable coordinate. Our free-end-point method for translations shows why the multiplier treatment is correct. The Maxwell action contains the velocity of an auxiliary variable but not the variable itself. It would be possible to start with a more general action assumed to depend on both an auxiliary and its velocity. The variation with respect to the auxiliary would then lead to a condition on the Lagrangian that is, in fact, satisfied by the Maxwell Lagrangian, which, as in the case of particle translations, does not depend on the auxiliary after all. As already noted, this is the case in all gauge theories hitherto studied. However, as this and the companion paper [1] show, scale-invariant theories necessarily contain both an auxiliary variable and its velocity. The free-end-point method shows that correct results are obtained by treating both as independent multipliers even though one is the velocity of the other.

The second comment is closely related to the first and relates to Dirac's classic study of generalized Hamiltonian dynamics [26]. He presents the consistent propagation of constraints derived from singular Lagrangians as more or less a matter of luck, which it certainly is in some cases 2]. However, as this paper and [1] show, best matching provides a transparent scheme in which constraints linear in the canonical momenta are automatically propagated because they reflect the existence of a geometrically defined metric on a quotient configuration space. The case of dilatational constraints provides a new and interesting example of consistent constraint propagation. Its generalization to geometrodynamics [1, which closely follows the treatment given here, leads to decidedly nontrivial relations.

\section{Scale-Invariant Dynamics with Specific Potentials}

We recall that the Jacobi action for a general Newtonian system is

$$
I_{\mathrm{Jacobi}}=2 \int \sqrt{E-U} \sqrt{\tilde{T}} \mathrm{~d} \lambda .
$$

Best matching with respect to translations and rotations restricts the Newtonian possibilities to a relatively limited extent. The total angular momentum must be zero and the potential $U$ must be a function of the inter-particle separations. Best matching with respect to dilatations has more far reaching consequences: the energy $E$ must necessarily be exactly zero and the potential must be homogeneous of degree -2 .

We first show how Newtonian gravity can be recovered to a good accuracy from a potential that is homogeneous of degree -2 . There are two easy ways to do this. Let

$$
W=\sum_{i<j} \frac{m_{i} m_{j}}{r_{i j}} .
$$

Thus, $W$ is minus the Newtonian gravitational potential with Newton's constant G set equal to unity. The first 'way to Newton' is to take as potential

$$
U=-\frac{W^{2}}{2}
$$

In the distinguished frame with the special $\lambda=t$, the equations of motion are

$$
\frac{\mathrm{d} \mathbf{p}^{i}}{\mathrm{~d} t}=-W \frac{\partial W}{\partial \mathbf{x}^{i}}
$$


This is Newton's law with G replaced by $W$. If the system has virialized and resembles a globular cluster, $W$ will remain effectively constant, and the motions will be essentially Newtonian. Astronomers will observe $1 / r$ forces, but the hidden dynamics will be scale invariant.

An island universe described by (32) can even mimic a Hubble-type expansion even though expansion has no physical meaning in scale-invariant dynamics. To see this, note that two processes can change $W$. The first is general expansion or contraction. Now this would change the moment of inertia (4),

$$
I=\frac{1}{M} \sum_{i<j} m_{i} m_{j} r_{i j}^{2}, \quad M=\sum_{i} m_{i},
$$

but the vanishing of the dilatational momentum $D$ enforces constancy of $I$. However, a changing shape of the matter distribution changes the scale-invariant Newtonian potential ${ }^{8}$

$$
U_{0}=-M \sqrt{I} W .
$$

The constancy of $I$ does not prevent the matter from 'clumping', which will increase both $U_{0}$ and $-W$, and with them the 'gravitational constant'. Suppose a planet orbiting a sun in an island universe that is becoming clumpier. In the distinguished frame, the gravitational forces will become stronger, and the planet-sun separation will adiabatically decrease. However, if we insist that, by definition, gravity is constant, this effect can be offset by an adiabatic increase of all scales from the distinguished frame. This will mimic a Hubble-type expansion.

In fact, although this is suggestive I doubt whether the Hubble red shift can be explained this way. The analogue of squaring $W$ in the conformal setting leads to a very complicated theory that may not even be consistent [1]. However, there is the much more interesting possibility that uses the unique conserved quantity in a scale-invariant universe, namely $I$, or rather $\sqrt{M I}=\mu$. This has a simple conformal analogue, which is the volume of 3 -space.

Therefore, to achieve scale invariance, and the strong equivalence principle, we shall use

$$
\mu=\sqrt{\sum_{i<j} m_{i} m_{j} r_{i j}^{2}} .
$$

Just as one passes from special to general relativity (with gravity minimally coupled to matter) by replacing ordinary derivatives in the matter Lagrangians by covariant derivatives, Newtonian potentials can be converted into potentials that respect scale invariance. One simply multiplies by an appropriate power of $\mu$, which has the dimensions of length. This is a rather obvious mechanism. What is perhaps unexpected is that the modified potentials lead to forces identical to the originals accompanied by a universal cosmological force with minute local effects. The scale invariance is hidden because $\mu$ is conserved.

Let $U$ consist of a sum of potentials $U_{k}$ each homogeneous of degree $k$ :

$$
U=\sum_{k=-\infty}^{\infty} a_{k} U_{k}
$$

The $a_{k}$ are freely disposable strength constants. The energy $E$ in the Jacobi action (8) will be treated as a constant potential $(k=0)$. (It plays a role like the cosmological constant $\Lambda$ in GR).

Now replace (35) by

$$
\tilde{U}=\sum_{k=-\infty}^{\infty} b_{k} U_{k} \mu^{-(2+k)}
$$

\footnotetext{
${ }^{8}$ The scale-invariant potential in fact determines all the qualitative properties of the Newtonian $N$-body problem and is a more important concept than the ordinary potential. For example, all asymptotic limiting motions of the 3-body problem terminate at one of the critical or singular points of $U_{0}$. They 'govern' the motion [30, 31].
} 
The equations of motion for (35) are

$$
\frac{\mathrm{d} \mathbf{p}^{i}}{\mathrm{~d} t}=-\sum_{k=-\infty}^{\infty} a_{k} \frac{\partial U_{k}}{\partial \mathbf{x}^{i}}
$$

for (36) they are

$$
\frac{\mathrm{d} \mathbf{p}^{i}}{\mathrm{~d} t}=-\sum_{k=-\infty}^{\infty} b_{k} \mu^{-(2+k)} \frac{\partial U_{k}}{\partial \mathbf{x}^{i}}+\sum_{k=-\infty}^{\infty}(2+k) b_{k} \mu^{-(2+k)} U_{k} \frac{1}{\mu} \frac{\partial \mu}{\partial \mathbf{x}^{i}}
$$

Since $\mu$ is constant 'on shell', we can define new strength constants that are truly constant:

$$
b_{k}=a_{k} \mu^{2+k} .
$$

The equations for the modified potential become

$$
\frac{\mathrm{d} \mathbf{p}^{i}}{\mathrm{~d} t}=-\sum_{k=-\infty}^{\infty} a_{k} \frac{\partial U_{k}}{\partial \mathbf{x}^{i}}+\sum_{k=-\infty}^{\infty}(2+k) a_{k} U_{k} \frac{1}{\mu} \frac{\partial \mu}{\partial \mathbf{x}^{i}} .
$$

If we define

$$
C(t)=\frac{\sum_{k=-\infty}^{\infty}(2+k) a_{k} U_{k}}{2 \sum_{i<j} m_{i} m_{j} r_{i j}^{2}}
$$

and express $\mu$ in terms of $r_{i j}$, then equations (38) become

$$
\frac{\mathrm{d} \mathbf{p}^{i}}{\mathrm{~d} t}=-\sum_{k=-\infty}^{\infty} a_{k} \frac{\partial U_{k}}{\partial \mathbf{x}^{i}}+C(t) \sum_{j} m_{i} m_{j} \frac{\partial r_{i j}^{2}}{\partial \mathbf{x}^{i}} .
$$

We recover the original forces exactly together with a universal force. It has an epoch-dependent strength constant $C(t)$ and gives rise to forces between all pairs of particles that, like gravitational forces, are proportional to the inertial mass but increase in strength linearly with the distance. ${ }^{9}$ The universal force will be attractive or repulsive depending on the sign of $C(t)$, which is an explicit function of the $r_{i j}$ 's. For small enough $r_{i j}$, they will be negligible compared with Newtonian gravity. An estimate will be made in section 7, but a first conclusion is this. A Newtonian-like universe with forces that appear to violate scaling may nevertheless be scale invariant with an as yet unrecognized cosmological force. Cosmologists might be greatly deceived, but, having once got the idea, they could predict the epoch-dependent $C(t)$ from the observed matter distribution and the locally observed forces.

Moreover, their revised cosmological model will be stable: its 'size', measured by $\mu$, remains constant - the automatic adjustment of $C(t)$ ensures it. Scale-invariant cosmology is free of the inescapable instability of Newtonian and Einsteinian cosmologies. What is more, $C(t)$ is unambiguously fixed by a fundamental symmetry, unlike Einstein's ad hoc $\Lambda$. It seems paradoxical that a theory constructed to eliminate size as a dynamical variable uses 'size' $(\mu)$ to generate a force that conserves the size (in the distinguished representation). Of course, the numerical value of the size is purely nominal. Moreover, the paradox is an artefact of the formalism. Could we but use shape coordinates conveniently, cosmology would be reduced to angles and mass ratios.

Note also that the 'size of the universe' is not kept constant because a repulsive cosmological force balances attractive gravity, as in Einstein's original cosmological model of 1917 [32]. That

\footnotetext{
${ }^{9}$ Although $C(t)$ is epoch dependent, this does not mean that the theory contains any fundamental coupling constants with such a dependence. The epoch dependence is an artefact of the decomposition of the forces into Newtonian-type forces and a residue, which is the cosmological force.
} 
achieved only unstable equilibrium. Scale-invariant cosmology is stable whatever the sign of $C(t)$. In Newtonian terms, the stability arises from the interplay of the potential's homogeneity and the simultaneous vanishing of the energy $E$ and the dilatational momentum $D$ (section 2). If one retains the same forces but allows the minutest deviation from zero of $E$ or $D$, the stability is lost, parabolically for $E \neq 0$ and linearly for $D \neq 0$. This again shows how cosmologists could be tricked. If they did not realize that a symmetry enforces stability, a scale-invariant cosmology would appear to be balanced on a knife edge. This is reminiscent of the 'flatness knife edge' of modern relativistic cosmology.

Before we obtain estimates, two potential flaws in the device of achieving homogeneity with powers of $\mu$ should be mentioned. The first is due to the possibility of collisions, which lead to infinities in the case of point particles. In Newtonian $N$-body theory, the most intractable collisions are the so-called central collisions in which all $N$ particles collide at once [33. They cannot be regularized and are rather like the Big-Bang or Big-Crunch singularities in GR. Both in Newtonian theory and in GR, the cosmological singularities clearly stem from the dynamical role of scale. Such singularities are not present in scale-invariant cosmology [1]. However, lesser collisions of $n<N$ particles are possible (and some form of gravitational collapse may also occur in conformal gravity). In the particle model, this will certainly cause $C(t)$ to become infinite even at a twoparticle collision, and the equations will be ill defined at such collisions. There is a similar potential problem in conformal gravity, and further research will be needed to establish whether it is serious. The second possible difficulty is related to dimensional analysis, to which we now come.

\section{Dimensional Analysis}

In Newtonian dynamics - and modern physics - there are three fundamental physical dimensions: mass $[m]$, length $[l]$, and time $[t]$. As long as no cosmological assumptions are made, these are necessarily independent and absolute, in the sense that one believes that 'they exist out there in the world'. One is forced to choose local units to measure them. If the universe is assumed to be self contained, the situation is different, because then the local unit is merely a fraction of some global total. More significantly, time can be eliminated as a dimension. One just has mass $[m]$ and length $[l]$. In scale-invariant geometrodynamics [1], dimensional analysis is even simpler and is based on length alone. The results obtained in this section are only the first step to this ideal. They are nevertheless suggestive. The method used here develops further the analysis of 34 .

In developing dimensional analysis, we consider two standpoints. The first is that of an imagined external observer who possesses a god's eye view of the universe. The second is that of an internal observer forced to use locally chosen units of mass, length, and time. The internal observer has no knowledge of the universe as a whole. For both observers, length $[l]$ is the most important dimension. Indeed, time is measured by length - the distance traversed by the hand of a clock - and masses are deduced from accelerations, which involve lengths and times. Let us therefore assume both kinds of observers are equipped with rods, on which they mark units of length. These rods can be used to measure the inter-particle separations $r_{i j}$. A measure of time $[t]$ is chosen by postulating that the motion of a particular body (the clock) measures time: when the clock traverses the already chosen unit of distance, one unit of time elapses. Finally, as Mach showed [12, relative masses can be deduced from the reciprocal ratio of the accelerations that bodies impart to each other when interacting in accordance with Newton's third law. If some given mass is chosen as the unit, other masses can then be determined in this way.

An extension of the same technique permits determination of charges. Since both masses and charges are deduced from accelerations, it seems to me that they should all have the dimension mass, denoted $[m]$. We shall see that, at least for gravitational and electrostatic forces, this leads to 
a consistent scheme in which the action principle of the universe contains no dimensional coupling constants but only passive mass (Newton's original inertial mass) and active charges: gravitational (which happen to be proportional to the passive mass), electrostatic and perhaps more. In standard dimensional analysis, the electric charge does not have the dimensions $[m]$ of mass, but it will in the proposed scheme. It then turns out (empirically) that the electric charge of the proton is $10^{19}$ times its gravitational charge (active gravitational mass), and both have dimension $[m]$. As we shall see at the end of this section, this assumption need not lead to conflict with the fact that the fine-structure constant $\alpha=e^{2} / h c$ is dimensionless because Planck's constant $h$ and the speed of light $c$ will have different dimensions.

Let us start by considering standard dimensional analysis in Newtonian theory for the case of gravitational and electrostatic interactions (of unit charges $\left|e_{i}\right|=e$ ). The Lagrangian is

$$
\sum_{i} m_{i} \frac{\mathrm{d} \mathbf{x}_{i}}{\mathrm{~d} t} \cdot \frac{\mathrm{d} \mathbf{x}_{i}}{\mathrm{~d} t}+\mathrm{G} \sum_{i<j} \frac{\bar{m}_{i} \bar{m}_{j}}{r_{i j}}-\sum_{i<j} \frac{e_{i} e_{j}}{r_{i j}}, \quad\left|\bar{e}_{i}\right|=\bar{e},
$$

where bars have been added to the masses in the gravitational potential to indicate that these are charges. Since the dimensions of the kinetic energy are taken as fiducial and are $m l^{2} t^{-2}$, it is clear that Newton's constant G must have dimensions $m^{-1} l^{3} t^{-2}$ if the gravitational charges are taken to have the dimensions of mass. It is equally clear that $e^{2}$ must have dimension $m l^{3} t^{-2}$. This can be achieved by setting

$$
\sum_{i<j} \frac{e_{i} e_{j}}{r_{i j}}=\mathrm{G} \sum_{i<j} \frac{\bar{e}_{i} \bar{e}_{j}}{r_{i j}}
$$

where $\bar{e} / \bar{m}$ is the ratio of the electrostatic force generated by unit electric charge to the gravitational force generated by the unit mass $\bar{m}$. For definiteness, I shall assume that there are $N=2 n$ particles of constant mass, $n$ of each carrying positive and negative unit electric charge. The total mass $M$ of the universe is equal to the sum of the inertial masses and equal to the sum of the gravitational and electrostatic charges, the contribution of the latter being in fact zero by the assumption of overall electric charge neutrality.

Let us now consider Jacobi's principle for Newtonian theory under the assumption that some hypothetical principle forces the energy $E$ to be exactly zero. The action can then be written in the form

$$
A=2 \sqrt{\mathrm{G}} \int \sqrt{\sum_{i<j} \frac{\bar{m}_{i} \bar{m}_{j}}{r_{i j}}-\sum_{i<j} \frac{\bar{e}_{i} \bar{e}_{j}}{r_{i j}}} \sqrt{\sum_{i} \frac{m_{i}}{2} \mathrm{~d} \mathbf{x}_{i} \cdot \mathrm{d} \mathbf{x}_{i}},
$$

which has the normal dimensions $m l^{2} t^{-1}$ of action: $[\sqrt{\mathrm{G}}]=m^{-1 / 2} l^{3 / 2} t^{-1}$ while [integrand] $=m^{3 / 2} l^{1 / 2}$. However, the constant $\sqrt{G}$ in front of the action $A$, being a common factor, can have no effect on the observed motions and can be simply omitted. Then the action $\bar{A}=A / \mathrm{G}$ in Jacobi's principle has dimensions $m^{3 / 2} l^{1 / 2}$. If a non-vanishing energy $E$ is included, it will have dimensions $m^{2} l^{-1}$, as is evident from (10). To recover the normal dimensions of energy, we must take the Newtonian time to have dimensions $m^{-1 / 2} l^{3 / 2}$. This is confirmed by the explicit expression (15) for the Newtonian time if, as above, we take the potential energy without $\mathrm{G}$ and therefore to have dimensions $m^{2} l^{-1}$.

Let us next consider the canonical momenta (11). They are homogeneous of degree zero in the velocities, and therefore any attempt to introduce a dimension of time into them will achieve nothing. They have the dimensions $m^{3 / 2} l^{-1 / 2}$. It is true that if we multiply the dimensions by $m^{-1 / 2} l^{3 / 2} / m^{-1 / 2} l^{3 / 2}=m^{-1 / 2} l^{3 / 2} / t$, we recover the Newtonian $m l t^{-1}$. However, this distorts the true nature of Jacobi geodesics, which involve only distances and masses.

We must now show how Newton's constant G is recovered as an emergent constant from the 
action $\bar{A}$. The Euler- Lagrange equations that follow from this action are

$$
\frac{\mathrm{d}}{\mathrm{d} \lambda}\left(\sqrt{\frac{\sum_{i<j} \frac{\bar{m}_{i} \bar{m}_{j}}{r_{i j}}-\sum_{i<j} \frac{\bar{e}_{i} \bar{e}_{j}}{r_{i j}}}{\sum_{i} \frac{m_{i}}{2} \frac{\mathrm{d} \mathbf{x}_{i}}{\mathrm{~d} \lambda} \cdot \frac{\mathrm{d} \mathbf{x}_{i}}{\mathrm{~d} \lambda}}} m_{i} \frac{\mathrm{d} \mathbf{x}_{i}}{\mathrm{~d} \lambda}\right)=-\sqrt{\frac{\sum_{i} \frac{m_{i}}{2} \frac{\mathrm{d} \mathbf{x}_{i}}{\mathrm{~d} \lambda} \cdot \frac{\mathrm{d} \mathbf{x}_{i}}{\mathrm{~d} \lambda}}{\sum_{i<j} \frac{\bar{m}_{i} \bar{m}_{j}}{r_{i j}}-\sum_{i<j} \frac{\bar{e}_{i} \bar{e}_{j}}{r_{i j}}}} \frac{\partial}{\partial \mathbf{x}_{i}}\left(\sum_{i<j} \frac{\bar{m}_{i} \bar{m}_{j}}{r_{i j}}-\sum_{i<j} \frac{\bar{e}_{i} \bar{e}_{j}}{r_{i j}}\right)
$$

In the discussion of Jacobi's principle, we simplified this equation by choosing the unique $\lambda$ that makes the square root on the right-hand side unity at all $\lambda$. In this way, we recovered Newtonian time. If this method is applied to (44), obtained from the action without G, the outcome can be interpreted in Newtonian terms as due to a choice of the time unit that makes $G=1$. Clearly, if we make the square root equal to a constant $k$, we simply recover a different time that runs uniformly w.r.t the first time but at a different rate. With this more general simplifying choice, (44) becomes

$$
m_{i} \frac{\mathrm{d}^{2} \mathbf{x}_{i}}{\mathrm{~d} t^{2}}=-k^{2} \frac{\partial}{\partial \mathbf{x}_{i}}\left(\sum_{i<j} \frac{\bar{m}_{i} \bar{m}_{j}}{r_{i j}}-\sum_{i<j} \frac{\bar{e}_{i} \bar{e}_{j}}{r_{i j}}\right)
$$

with 'gravitational constant'

$$
k^{2}=\frac{\sum_{i} \frac{m_{i}}{2} \frac{\mathrm{d} \mathbf{x}_{i}}{\mathrm{~d} t} \cdot \frac{\mathrm{d} \mathbf{x}_{i}}{\mathrm{~d} t}}{\sum_{i<j} \frac{\bar{m}_{i} \bar{m}_{j}}{r_{i j}}-\sum_{i<j} \frac{\bar{e}_{i} \bar{e}_{j}}{r_{i j}}}
$$

which has the correct dimensions $m^{-1} l^{3} t^{-2}$. Let us now recall our internal observers within such a universe, who have chosen arbitrarily units of mass, length, and time and use standard Newtonian dimensional analysis. Making purely local observations within their solar system, they will find the the gravitational constant has some value G. Making cosmological observations, they will discover that $\mathrm{G}$ has the value $k^{2}(46)$. Since they include $\mathrm{G}\left(=k^{2}\right)$ as a factor in the potential energy, they will interpret (46) as saying that minus the Newtonian potential energy is equal to the kinetic energy. Thus, they will find that the total energy of the universe is exactly zero and will interpret this as a 'cosmic coincidence' since they can find no theoretical argument that enforces the condition.

From the point of view of a dynamics of pure shape, Newtonian theory in Jacobi form is clearly imperfect, this being reflected in the fact that the action depends on the length scale. Also, no fundamental principle justifies the assumption that $E=0$. However, the dependence on the mass scale is not serious, because all masses are constant and can be expressed as dimensionless ratios of the total mass $M$.

Let us now repeat the dimensional analysis and 'calculation of $G$ ' for scale-invariant gravity (SIG) and electrostatics with the assumption that the necessary homogeneity of degree -2 of the potential is achieved as above by means of $\mu$. This is a much more interesting theory because both the energy and the dilatational momentum must be exactly zero.

As is evident from (45), the basic form of the equation of motion for Jacobi-type actions (with $E=0$ either by stipulation or enforced by scale invariance) is

$$
m_{i} \frac{\mathrm{d}^{2} \mathbf{x}_{i}}{\mathrm{~d} t^{2}}=-\frac{T}{U} \frac{\partial U}{\partial \mathbf{x}_{i}}
$$

with $k^{2}=T / U$ identified as Newton's constant. To pass from Newtonian theory to scale-invariant gravity, we simply replace the Newtonian potential $U$ by $U / \mu$. Then the equation of motion becomes

$$
m_{i} \frac{\mathrm{d}^{2} \mathbf{x}_{i}}{\mathrm{~d} t^{2}}=-\frac{T \mu}{U} \frac{\partial}{\partial \mathbf{x}_{i}}\left(\frac{U}{\mu}\right)=-\frac{T}{U} \frac{\partial U}{\partial \mathbf{x}_{i}}+\frac{T}{\mu} \frac{\partial \mu}{\partial \mathbf{x}_{i}} .
$$


We have the identical expression $T / U$ for $\mathrm{G}$, but there is now a more subtle reason for its constancy. First, $T \mu / U$ is made constant by choosing one of the distinguished times. This is the device that made $T / U=\mathrm{G}$ constant in the Newtonian case. Second, $\mu$ is constant in scale-invariant gravity as a consequence of the dynamics. Thus, in (47) the coefficient of the gravitational force is constant because of these two reasons together. The coefficient of the cosmological force is epoch dependent. Both coupling constants are emergent and uniquely determined. We shall estimate them in the next section. The strength of electrostatics relative to gravity is constant and, in the absence of unification of the forces of nature, must be determined empirically.

Two possible problems in scale-invariant gravity were noted at the end of the previous section. The one to do with dimensional analysis concerns the extension of the method presented in this section to Newtonian forces with other than $1 / r$ distance dependence. Presumably, one will always want the dimensions of all terms in the potential to be the same. For gravity and electrostatics, the dimensions are $m l^{-2}$, but it seems to me that it will be difficult to find the right combination of charges to give these dimensions for other than $1 / r$ Newtonian forces without introducing undetermined constants with dimensions of mass. This may not be a problem or, if it is, it might even be a virtue through ruling out such forces, which are not observed. However, I think the particle model is probably not a reliable guide for such arguments. At the least, there is no problem with gravity and electrostatics.

Continuing with the dimensional analysis, calculation of the various dimensions of the most important dynamical quantities for scale-invariant gravity (SIG) together with the dimensions for standard Newtonian theory (SNT) and 'Jacobianized' Newtonian theory (JNT) as calculated earlier gives the following results:

\begin{tabular}{cccccc}
\multicolumn{2}{c}{ Action } & Length & Momenta & Time & Energy \\
SNT & $m l^{2} l^{-1}$ & $l$ & $m l t^{-1}$ & $t$ & $m l^{2} t^{-2}$ \\
JNT & $m^{3 / 2} l^{1 / 2}$ & $l$ & $m^{3 / 2} l^{-1 / 2}$ & $m^{-1 / 2} l^{3 / 2}$ & $m^{2} l^{-1}$ \\
SIG & $m$ & $l$ & $m l^{-1}$ & $l^{2}$ & $m l^{-2}$
\end{tabular}

The results for the scale-invariant case are striking and suggestive. First, since mass is a ratio, the action is effectively dimensionless. This suggests that for a scale-invariant theory the quantum of action will be a pure number. A similar calculation for scale-invariant geometrodynamics also suggests that action is a pure number. Also remarkable are the dimensions of the canonical momenta, which exactly mirror the de Broglie relation for the momentum in quantum mechanics. It is worth emphasizing that these results are non-trivial and arise from a 'trinity' of requirements: no time, scale invariance, and the implementation of the strong equivalence principle by the use of $\mu$.

The at first enigmatic result that in the scale-invariant case 'time is an area' is very natural. One of the length dimensions arises from the fact that the hand of clock must traverse a length to measure time. This length is supplied by the particle displacements in the numerator of (15). The other length comes from the weighting by the denominator of (15) and the fact that scale invariance and the SEP require the potential to have dimensions $m l^{-2}$.

Let us now consider what happens to the fine-structure constant by applying the rule for passing from the Newtonian dimensions $m, l, t$ to their scale-invariant timeless counterparts, which is simply to replace $t$ by $l^{2}$ and remember that the Newtonian potentials have been divided by $\mu$. Thus, the Newtonian dimensions of velocity are $[v]=l t^{-1}$, but now $[v]=l^{-1}$. Similarly, the Newtonian dimensions of Planck's constant are $[h]=m l^{2} t^{-1}$, but now they become $[h]=m$, as we have already found. Thus, the $h c$ that appears as the denominator of the fine-structure constant no 
longer has its Newtonian dimensions $m l^{3} t^{-2}$ but $m l^{-1}$. Let us now consider the electric charge $e$. In Newtonian mechanics, it appears in the potential $\psi=\sum_{i<j} e_{i} e_{j} / r_{i j}$, but in the new scheme this is replaced by $\psi \mu^{-1}$. In Newtonian mechanics, $e^{2} / h c$ is dimensionless, so $\left[e^{2}\right]=m l^{3} t^{-2}$. However, if our approach is on the right track, the quantity that hitherto has been taken to be $e^{2}$ is actually $e^{2} \mu^{-1}$, and $[\mu]=m l$. Therefore, if we postulate $[e]=m$, the new dimensions of $e^{2}$ are $m l^{-1}$, which is what we just found for $[h c]$, so the 'new fine-structure constant' is still dimensionless. Even though gravitational charge does not appear to be quantized in the way electric charge is, one can still define a 'gravitational fine-structure constant', which will also be dimensionless.

Only if and when a scale-invariant quantum cosmology has been created will it be possible to say whether the relations found in this section have a deep meaning or are merely fortuitously suggestive. However, there must surely be an a priori presumption that scale invariance will overturn current ideas about the fundamental constants of nature. When Planck [35] deduced his famous units of length, time, and mass, he employed $h, c, G$. He noted that the units will have fundamental significance so long as the theories from which they are deduced are correct. The analysis of this section suggests that, if the universe is self contained and scale invariant, the quantum of action is a pure number. If so, it seems inevitable that a reorientation of our ideas about dimensional analysis and the constants of nature will be necessary.

\section{Estimates}

Let us make estimates for the model with gravity and electrostatics. Suppose an island universe of $N$ point particles half of which carry positive unit charges $e_{i}$ and the other half negative charges $-e_{i}=-1$ ( $N$ is an even integer). With the notation of the previous section, take the Jacobi-type action to be

$$
I_{\text {Model }}=\int \mathrm{d} \lambda \sqrt{\frac{E}{\mu^{2}}+\frac{1}{\mu} \sum_{i<j} \frac{\bar{m}_{i} \bar{m}_{j}}{r_{i j}}-\frac{1}{\mu} \sum_{i<j} \frac{\bar{e}_{i} \bar{e}_{j}}{r_{i j}}} \sqrt{T}=\int \mathrm{d} \lambda \sqrt{P} \sqrt{T},
$$

where $T$ is best matched.

Although no principle forbids the 'energy' term $E / \mu^{2}$ in the potential, it will merely lead to a further cosmological force that adds no interest and introduces the undetermined constant $E$ (with dimension $[m]$ ). Let us therefore omit it, and hope that eventually some principle will rule it out - and determine the electrostatics-to-gravity strength ratio $\bar{e} / \bar{m}$, which for the moment must be fixed empirically. Let us make estimates using $\bar{e}^{2} / \bar{m}^{2} \approx 10^{40}$, reflecting the relative strengths of the electrostatic and gravitational interactions for the common elementary particles. Let us also take $N=10^{80}$ particles, since this is the estimated number of nucleons in the observed universe within the Hubble radius $H$. For simplicity, let us take all masses $\bar{m}_{i}=\bar{m}$ with $\bar{m} N=M$.

As cosmological model, we first assume a spherically symmetric and more or less uniform 'plasma' matter distribution like a globular cluster with mean radius $R$. In the estimation of (minus) the potentials

$$
\mu, \quad \psi=\sum_{i<j} \frac{\bar{m}_{i} \bar{m}_{j}}{r_{i j}}, \quad \phi=-\sum_{i<j} \frac{\bar{e}_{i} \bar{e}_{j}}{r_{i j}},
$$

the fact that all masses are positive while half the charges are positive and half negative leads to big differences. Both $\mu$ and $\psi$ contain $\approx N^{2}$ terms formed by all pairs of particles, for which the mean separation will be $R$. Each mass 'feels' all the other $N-1$ masses, and the mean contribution of each pair will be $\bar{m}^{2} / R$. In contrast, each charge $\bar{e}_{i}$ finds itself in an almost exactly neutral cloud. In fact, the total charge outside any considered charge $\bar{e}_{i}$ will be $-\bar{e}_{i}$, and the 'mean distance' of the opposite charge will be $R$. Therefore, to a good accuracy the electrostatic potential $\phi$ consists 
of $N$ terms, each of magnitude $\approx \bar{e}^{2} / R .^{10}$ (The minus has disappeared because the charges are opposite.)

Therefore, in the 'plasma' state

$$
\mu \approx N \bar{m} R, \quad \psi \approx \frac{N^{2} \bar{m}^{2}}{R}, \quad \phi \approx \frac{N \bar{e}^{2}}{R},
$$

and the corresponding contributions to $P$ in (48) are

$$
P_{\text {plasma }}=\frac{\psi}{\mu}+\frac{\phi}{\mu} \approx \frac{N \bar{m}}{R^{2}}+\frac{10^{40}}{R^{2}}, \quad \bar{m}=1,
$$

so that for $N \sim 10^{80}$ the (positive) electrostatic contribution to $P$ will be only $10^{-40}$ of gravity's.

Now suppose the 'plasma' evolves into a 'post-nucleosynthesis' state in which all the charges are in $N / 2$ neutral pairs with inter-charge separation $\bar{r}$. This pairing will change $\phi$ to $\phi \approx-N \bar{e}^{2} / \bar{r}$, have negligible effect on $\mu$ and will only change $\psi$ significantly if $\bar{r} \leq 10^{-80} R$. The possibility of 'gravitational collapse' to such an extremely small relative size, which corresponds to $\sim 10^{-20} l_{\text {Planck }}$ for $R \sim 10^{28} \mathrm{~cm}$, will not be considered here. Instead, let us consider the more immediately realistic case $\bar{r} / R \sim 10^{-40}$, so that $\bar{r}$ is of nuclear order $10^{-12} \mathrm{~cm}$. Then, in contrast to the 'plasma' estimate (50), we obtain the 'post-nucleosynthesis' estimate

$$
P_{\mathrm{pns}}=\frac{\psi}{\mu}-\frac{\phi}{\mu} \approx \frac{N \bar{m}}{R^{2}}+\frac{10^{40}}{R \bar{r}},
$$

so now the two contributions will have equal orders of magnitude.

Making the assumption that the particle model can give valid order-of-magnitude estimates, let us now try to estimate the size and mass of the actual universe. In accordance with the above estimates, let us assume that the combined potential $U$ of the gravitational and electrostatic potentials is $\approx M^{2} / R$. Then

$$
G=\frac{T}{U}=\frac{R \sum m_{i} \sum_{i} \frac{\mathrm{d} \mathbf{x}_{i}}{\mathrm{~d} t} \cdot \frac{\mathrm{d} \mathbf{x}_{i}}{\mathrm{~d} t}}{M^{2}} .
$$

To extract useful information from this, it is necessary to identify the characteristic values of the velocities that appear in the numerator. Since special relativity prohibits velocities above the speed of light $c$, while relativistic speeds are realized in nuclei, let us assume for simplicity that all velocities are $\approx c$. Then

$$
G \approx \frac{R M c^{2}}{M^{2}} \approx \frac{c^{2}}{\rho R^{2}}
$$

where $\rho$ is the matter density, assumed constant. Since $G$ and $c$ are known accurately, and $\rho$ is known approximately, we can estimate the 'radius of the universe':

$$
R \approx \sqrt{\frac{c^{2}}{G \rho}} .
$$

Taking $\rho \approx 10^{-30}$ g.cm ${ }^{-3}$, and substituting the known values of $c$ and $G$, we find

$$
R \approx 10^{29} \mathrm{~cm}, \quad M \approx 10^{57} \mathrm{~g} .
$$

\footnotetext{
${ }^{10}$ Physicists wonder how it will ever be possible to explain the huge difference between the strength of gravity and the remaining forces of nature. It seems to me not impossible (see the first footnote on p. 23 of [34]) that, in a self-contained universe, it is somehow a consequence of the very different ways in which the potentials for gravity and electrostatics are calculated. This might have far-reaching consequences in quantum cosmology.
} 
Such relationships are obtained in modern cosmology, in which they reflect the fact that the universe has a near-critical density and is therefore spatially flat. The estimates for the radius and mass of the universe refer to the current epoch and increase with time. Inflation is invoked to explain the flatness, which must otherwise be regarded as a cosmic coincidence. In scale-invariant gravity, the interpretation is different. An analogue of the flatness coincidence is a prediction that follows from the basic dynamical structure of the theory. Moreover, the radius $R$ of the universe cannot change significantly because $\mu \approx M R$ is constant. Of course, $R$ has no absolute meaning. The significance of the above estimates is that they relate human dimensions to the cosmic dimensions. The issue of whether scale-invariant gravity has any chance of replacing the standard hot big-bang model will be discussed in the next section. In this section, it only remains to estimate the relative strengths of gravity and the cosmological force.

Since $\mathbf{F}$ increases linearly with the distance $r$ while gravity decreases as $1 / r^{2}$, the relative strengths of the two forces will depend on $r$. The simple calculation shows, first, that the cosmological force must be attractive if the only Newtonian forces are gravity and electrostatics ${ }^{11}$ and, second, that at distance $\tilde{r}$ the ratio of $\mathbf{F}$ to gravity is

$$
\frac{\text { cosmological force }}{\text { gravitational force }} \sim \frac{r^{3}}{R^{3}} .
$$

This result can be expected to survive in conformal gravity. It shows that on solar-system scales, for which $r / R \sim 10^{-14}$, the effect of $\mathbf{F}$ will be $\sim 10^{-42}$ and negligible. However, for $r \sim R$, the two forces will have the same magnitude. The present derivation of the cosmological force as a scaling concomitant of gravity (and possibly other forces in certain epochs) differs markedly from all ad hoc introductions of a cosmological constant: $\mathbf{F}$ must occur, its strength is fixed and extremely weak within the solar system, and it is always 'tuned' to maintain a universe of constant size. ${ }^{12}$ The relative strength of $\mathbf{F}$ may increase in a 'post-nucleosynthesis' phase.

\section{An Alternative Cosmology?}

It seems barely possible that scale-invariant gravity could replace GR and give a viable cosmology. The standard hot Big Bang scenario looks very secure. However, scale invariance is an attractive principle, and the manner in which GR just fails to be scale invariant (spelled out in [1]) is mysterious. Moreover, it is precisely this 'failure' that makes an expanding universe possible. For this reason, the best-matching scheme, with its definite and striking predictions (551) and (56), can, at the least, act as a useful foil to the current paradigm.

In this spirit, I shall merely make a few comments. Serious conclusions must await detailed calculations using conformal gravity, the structure of which is now settled [1]. However, it looks as if the first obvious test will be passed easily: as [1] shows, conformal gravity at human, solar-system and binary-pulsar mass and distance scales appears to agree with GR as well as the scale-invariant (48) agrees with Newtonian gravity. The real problems are all in cosmology, in which the major difference between the two theories is this. In the standard model the universe is simultaneously expanding and becoming steadily more inhomogeneous. In the scale-invariant case, the universe cannot expand, since that has no meaning. It can only become more inhomogeneous - it can only 'change its shape'.

\footnotetext{
${ }^{11}$ The expressions (38) and (39) simplify if there is only one value of $k$, and we can use the equation (47).

${ }^{12}$ One can see qualitatively how this happens. Suppose a particle in a 'cloud' that forms an island universe manages to escape a significant distance from the cloud. It will then feel a restoring force that grows linearly with distance and will be rapidly pulled back into the cloud as if it were on a piece of elastic. Similarly, if the cloud becomes egg shaped, the particles at the ends of the long axis will feel an enhanced restoring force.
} 
Now it is a fact that, while remaining isotropic and homogeneous on large scales, the presently observed universe is obviously hugely more structured than it was in the past. It has changed its shape massively. The estimates of section 7 show how readily the scale-invariant potential energy can increase if the universe becomes more clumpy. Scale-invariant gravity must, in the first place, yield a cause of the Hubble red shift. The only plausible candidate that I can see is this change in the 'potential' of the universe induced by such clumping. It is suitably great and, according to the standard model, has been happening since the end of inflation. Therefore, the conjecture has to be that somehow the change in potential causes the Hubble red shift. This is not inherently impossible. We know that differences in the gravitational potential give rise to a gravitational red shift. Moreover, in scale-invariant gravity the increases in all forms of potential energy increase the cosmological force, which, being universal and acting on mass, is gravitational in nature. Therefore, it seems to me not entirely impossible that clumping could give rise to some kind of gravitational red shift. In the realm of classical physics, the integrated Sachs-Wolfe [36] effect would certainly be relatively more important than it is in Einsteinian cosmology. However, it seems to me that it must still fall far short of what is needed. Possibly more relevant is the fact that, in scale-invariant gravity, the cosmological force mimics a negative cosmological constant whose strength may have increased by orders of magnitude over cosmological epochs. Its impact on galactic dynamics is obviously a matter of some interest, as is the possibility that it might somehow have a bearing on the red shift. For the moment, I do not think that anything more can be usefully said about this crucial issue, except perhaps that scale-invariant gravity does force one to look for totally new explanations for fundamental effects. That cannot be bad. In the next section, I shall mention possible quantum effects induced by clumping.

At this stage, it is premature to try to attack detailed issues such as primordial nucleosynthesis and the origin of the microwave background. However, the isotropy and homogeneity of the observed universe on large scales and the problem of singularities seem to me to much more promising topics. I shall discuss them in the context of particle dynamics.

In considering these issues, the difference between the configuration spaces $\mathrm{Q}$ and $\mathrm{Q}_{0}$ for theories with and without scale invariance is significant. Consider the Newtonian gravitational $N$-body problem for $N \geq 3$. It is dominated by the uniquely distinguished point in $\mathrm{Q}$ at which all particles coincide. In 25] I have called this point (and its analogues in other configuration spaces) Alpha. The singularity of both the topology and the potential at Alpha causes the unregularizable central collisions mentioned earlier. But Alpha is not a 'shape' and does not belong to shape space $\mathrm{Q}_{0}$ [37. However, $Q_{0}$ has a rather different uniquely distinguished configuration Alpha . It is the most uniform state, at which the (negative) scale-invariant potential has an absolute maximum. In the 3- and 4-body problems, this is at the equilateral triangle and quadrilateral, respectively, whatever the masses of the particles [30]. The topology, potential and scale-invariant dynamics are very well behaved at Alpha $a_{0}$. There cannot be anything like the Big-Bang singularity.

There is another difference. The dynamical curve of the Newtonian $N$-body problem can enter (or leave) Alpha with arbitrary energy $E$ and dilatational momentum $D$ (but only with vanishing angular momentum). If emergence from Alpha is seen as a model Big Bang, the statistics of initial states must include a spectrum of $E$ and $D$ values. In contrast, $E=D=0$ in scale-invariant dynamics. Intuitively, the fulfilment of the constraint requiring the dilatational momentum to vanish, especially in conformal gravity, where it becomes a constraint at each space point, must favour isotropic momenta distributions. At the very least, the undoubted existence of the smooth absolute maximum of the scale-invariant potential at the most uniform state must be significant. The critical points of the potential always exert a decisive influence on the classical [31, 33] and quantum 38. physical processes that unfold in a configuration space. I believe scale invariance opens up new possibilities for explaining (without fine tuning and the need to postulate as yet 
unknown scalar fields) those features of the universe for which inflation is currently invoked.

As a final example, let me mention the flatness problem. The current theory is unable to explain why the universe seems to be expanding at almost exactly its escape velocity. In Newtonian terms, this corresponds to expansion with total energy $E$ exactly equal to zero. In the standard theory, there is no fundamental principle that can enforce $E=0$. However, we have seen that this is exactly what scale invariance does do. Of course, at the same time, it rules out expansion, predicting, in particle dynamics, a universe like a globular cluster. Nevertheless, there is a principle that enforces $E=0$.

To conclude this tentative discussion of classical cosmology, scale invariance encourages radical questioning of accepted ideas and opens up some interesting possibilities.

\section{Possible Quantum Implications}

The dimensional analysis discussed in section 6 indicates that if the universe is described by a scale-invariant law it will be necessary to revise our ideas about the constants of nature. Section 6 shows that it is in principle possible to calculate Newton's constant G in a scale-invariant framework and suggests that the quantum of action does not have dimensions $m l^{2} t^{-1}$ but is a pure number. These major changes are a consequence of the elimination of time from kinematics, the transition to potentials that are homogeneous of degree -2 , and the inclusion of $\mu$, the square root of the moment of inertia of the universe, in the fundamental action principle.

There is another very direct way of seeing that potentials homogeneous of degree -2 may well have far-reaching consequences. The need for such potentials is a direct consequence of having a kinetic energy quadratic in the velocities. Each velocity has the dimension of length (divided by time), and this is why we need the degree -2 in the potential. Now the quadratic kinetic energy of classical physics leads to the Laplacian in quantum physics. If one solves the time-independent Schrödinger equation for the $1 / r$ electrostatic potential, there is a mismatch between the dimensions of the potential and the Laplacian. A constant with the dimensions of length is needed. It seems to me that this is the origin of the Planck length. A scale-invariant time-independent Schrödinger equation would need no such constant - the dimensions match without one.

The canonical approach to quantum gravity leads to the Wheeler-DeWitt equation 39], which is very like the time-independent Schrödinger equation [24, 25]. In the case of conformal gravity, it will not contain any dimensional constant. In the naivest approach, one will expect a solution of the equation, if it exists, to give (static) probabilities for different possible shapes of the configuration of the universe. In the case of the scale-invariant 3-body problem, one should then obtain probabilities for shapes of triangles. Since the scale-invariant potential for the 3-body problem has infinitely deep wells at the two-body coincidences and wave functions tend to collect in potential wells, it could well be that shapes with one short side of the triangle and two long ones will get the highest probabilities. These correspond, of course, to configurations close to a two-particle coincidence. If so, I would see this as an embryonic theoretical derivation of the fundamental ratio of scaleinvariant quantum cosmology, namely the ratio of the shortest to the longest length realized in the most probable configurations. There cannot be a Planck length and a Hubble radius, but only a shortest-to-longest ratio. It is therefore possible that radical scale invariance as proposed in this paper and in [1] has the potential to derive the Planck length from first principles.

This may also have a bearing on the Hubble red shift. Since it is revealed through quantum transitions, a complete explanation of it must be based on a quantum theory of the universe. This theory is likely to be strongly affected by the key role that the total potential (both gravitational and non-gravitational) plays in the classical theory - in the 'formula for time' (15) it determines how much a given amount of change 'advances time' and in (26) it is a factor in the canonical momenta. 
The potential is therefore likely to have a strong effect on the relative sizes and structures of quantum objects such as atoms and molecules, but these sizes themselves determine the potential. In the classical scale-invariant theory, the nominal initial size of the universe is conserved by the classical equations in the distinguished representation. However, without a quantum form of the theory, we cannot say what we shall measure using physical rods and clocks. Given the central role of the potential in all the basic equations of the theory, it is at least possible that it would affect rods, making them relatively shorter in states of the universe with high absolute magnitude of the potential. This would then mimic an apparent expansion of a universe that becomes more clumpy.

Despite the tentative and speculative nature of these two final sections, I hope they will serve to emphasize the potential importance of scale invariance. Weyl's Cartesian dream represents unfinished business.

Acknowledgements. I have benefitted greatly from discussions with the $N$-body specialists Alain Albouy, Alain Chenciner, Jörg Elsner, Douglas Heggie, Piet Hut, Christian Marchal, Richard Montgomery, and Carles Simó. Richard suggested the use of the moment of inertia to achieve the necessary homogeneity [40] and the Alains and Carles showed me what could be done with the Lagrange-Jacobi relation. Ted Jacobson also helped in the early stages of this work. I thank John Briggs for discussion of quantum aspects. I have also had much help from my collaborators Edward Anderson, Brendan Foster, and Niall Ó Murchadha. The notion of the corrected coordinate is due in large part to Brendan. I also thank a referee and a member of the editorial board for helpful suggestions for improvement of the first draft.

\section{Appendix: Hamiltonian Formulation}

As Lanczos explains [19, p. 169, the Hamiltonian formalism in variational mechanics is really a special case of the Lagrangian method for which the kinetic energy has a particularly simple (canonical) form, namely $T=\sum p^{i} \dot{q}_{i}$. Thus, the (first-order) Lagrangian of the Hamiltonian method is

$$
A=\int \mathrm{d} t\left[\sum p^{i} \dot{q}_{i}-H\left(q_{1}, \ldots, q_{n} ; p^{1}, \ldots, p^{n}\right)\right],
$$

where $H$ is the normal Hamiltonian and is treated for variational purposes as if it were a potential.

From the Lagrangian (57), in which the $p^{i}$ 's are coordinates on an equal footing with the $q_{i}$ 's except that they appear as Lagrange multipliers (their velocities do not occur in the Lagrangian, unlike the $\dot{q}_{i}$ 's), one calculates the Euler-Lagrange equations in the standard manner. They turn out to be the Hamiltonian equations.

To obtain a Hamiltonian treatment appropriate for scale-invariant gravity, two modifications to the standard treatment are needed. First, the Lagrange function of any Jacobi action is homogeneous of degree one in the velocities. This leads to the identity (12), which in turn has the consequence that when one attempts to calculate the Hamiltonian by the rule $H=p^{i} \dot{q}_{i}-\mathcal{L}$ an expression that vanishes identically is obtained. This problem is overcome in Dirac's generalized Hamiltonian theory [26] by converting (12) into the quadratic constraint

$$
\mathcal{H}=\sum_{i} \frac{\mathbf{p}_{i} \cdot \mathbf{p}_{i}}{2 m_{i}}-(E-U)=0
$$

and taking $H=N \mathcal{H}$, where $N$ is a Lagrange multiplier, as the Hamiltonian. In the scale-invariant case, the constraint is of the same basic form but, first, $E=0$ and, second, $\overline{\mathcal{L}}$ is a function of the corrected coordinates $\bar{q}_{i}$ and their velocities. The quadratic constraint (in the one-dimensional case) is

$$
\overline{\mathcal{H}}=\sum_{i} \frac{p^{i} p^{i}}{2 m_{i}}+\left(a^{\mathrm{d}}\right)^{2} U\left(\bar{q}^{i}\right)=0, \quad \bar{q}^{i}=a^{\mathrm{d}}\left(q^{i}+a^{\mathrm{t}}\right),
$$


where $a^{\mathrm{t}}$ and $a^{\mathrm{d}}$ are the gauge variables corresponding to translations and dilatations, and the $\left(a^{\mathrm{d}}\right)^{2}$ appears in the constraint because $p^{i}=a^{\mathrm{d}} \partial \overline{\mathcal{L}} / \partial v^{i}$. Any other constraints obtained in the Lagrangian treatment must be added with further Lagrange multipliers. In our case, we shall need to add, with appropriate multipliers, the primary constraints (27) corresponding to the various types of best matching. This is relatively standard, through there is, as we shall shortly see, a slight departure from Dirac's procedure. The second modification concerns the treatment of the gauge auxiliary variables. We need to find a Hamiltonian treatment that corresponds to the freeend-point variation in the Lagrangian approach. Both modifications are achieved if we use the first-order principle

$$
A=\int \mathrm{d} \lambda\left(\sum_{i} p^{i} \dot{q}^{i}+p^{\mathrm{t}} \dot{a}^{\mathrm{t}}+p^{\mathrm{d}} \dot{a}^{\mathrm{d}}-N \overline{\mathcal{H}}-N^{\mathrm{t}}\left(p^{\mathrm{t}}-\sum_{i} p^{i}\right)-N^{\mathrm{d}}\left(p^{\mathrm{d}} a^{\mathrm{d}}-\sum_{i} p^{i} q^{i}\right)\right),
$$

where $p^{\mathrm{t}}, p^{\mathrm{d}}$ are the canonical momenta corresponding to auxiliary gauge variables, and $N^{\mathrm{t}}, N^{\mathrm{d}}$ are multipliers associated with the translational and dilatational constraints.

If we now vary (58) with respect to the multipliers $N, N^{\mathrm{t}}, N^{\mathrm{d}}$, we obtain

$$
\overline{\mathcal{H}}=\sum_{i} \frac{p^{i} p^{i}}{2 m_{i}}+\left(a^{\mathrm{d}}\right)^{2} U\left(\bar{q}^{i}\right)=0, \quad p^{\mathrm{t}}-\sum_{i} p^{i}=0, \quad p^{\mathrm{d}} a^{\mathrm{d}}-\sum_{i} p^{i} q^{i}=0 .
$$

We now employ the free-end-point method and require the variation of the action w.r.t both the coordinates $a^{\mathrm{t}}, a^{\mathrm{d}}$ and the velocities $\dot{a}^{\mathrm{t}}, \dot{a}^{\mathrm{d}}$ of the auxiliary gauge variables to vanish. The variation w.r.t the velocities tells us

$$
p^{\mathrm{t}}=0, \quad p^{\mathrm{d}}=0
$$

and in conjunction with (59) these imply

$$
\sum_{i} p^{i}=0, \quad \sum_{i} p^{i} q^{i}=0
$$

Variation with respect to the auxiliary variables $a^{\mathrm{t}}$ and $a^{\mathrm{d}}$ gives

$$
\frac{\partial U}{\partial a^{\mathrm{t}}}=-\sum_{i} \frac{\partial U}{\partial q^{i}}=0, \quad p^{\mathrm{d}}+2 a^{\mathrm{d}} U+\left(a^{\mathrm{d}}\right)^{2} \frac{\partial U}{\partial a^{\mathrm{d}}}=0
$$

The first of these conditions is the one that ensures Newton's third law, and, since we already have $p^{\mathrm{d}}=0$, the second gives the homogeneity condition on the potential.

Since the momenta are regarded as independent variables, we also vary w.r.t the momenta of the auxiliary variables, which gives

$$
\dot{a}^{\mathrm{t}}=N^{\mathrm{t}}, \quad \dot{a}^{\mathrm{d}}=N^{\mathrm{d}} .
$$

Finally, Hamilton's equations are

$$
\dot{q}^{i}=\frac{\partial H}{\partial p^{i}}=N \frac{p^{i}}{m_{i}}+N^{\mathrm{t}}+N^{\mathrm{d}} q^{i}, \quad \dot{p}^{i}=-N\left(a^{\mathrm{d}}\right)^{2} \frac{\partial U}{\partial q^{i}}+N^{\mathrm{d}} p^{i} .
$$

These reduce to Newton's equations if we go over to the distinguished representation by setting $N=1$ and $N^{\mathrm{t}}=N^{\mathrm{d}}=0$. However, we can perfectly well specify the three multipliers freely. Then $N^{\mathrm{t}}$ and $N^{\mathrm{d}}$ are velocities in the unphysical gauge directions generated by translations and dilatations as in Dirac's treatment [26, and $N$ is the unphysical 'label speed' at which we move along the dynamical orbit in shape space. 
However, it will be noted that we have generalized Dirac's treatment by the inclusion of auxiliary gauge variables in addition to the conventional physical variables and multipliers. The simpler and more familiar form given by Dirac corresponds to the replacement of (158) by

$$
A^{*}=\int \mathrm{d} \lambda\left(\sum_{i} p^{i} \dot{q}^{i}-N\left(\sum_{i} \frac{p^{i} p^{i}}{2 m_{i}}+\left(a^{\mathrm{d}}\right)^{2} U\left(\bar{q}^{i}\right)\right)-N^{\mathrm{t}} \sum_{i} p^{i}-N^{\mathrm{d}} \sum_{i} p^{i} q^{i}\right) .
$$

Variation w.r.t the multipliers $N, N^{\mathrm{t}}, N^{\mathrm{d}}$ gives the first member of (159) and (60). However, the first member of (61) no longer arises from the variational principle but must be adjoined if the constraint $\sum_{i} p^{i}=0$ is to propagate. In addition, the status of $a^{\mathrm{d}}$ is strange. Variation w.r.t it does give the second member of (61), but (62) has no variational derivation and its second member must be adjoined in order to propagate the constraint $\overline{\mathcal{H}}=0$. We see that in the presence of scale invariance the Dirac procedure works but lacks a transparent variational basis.

More could be said about these matters, above all about a new unifying perspective on gauge theory that best matching provides. But since this is already a long paper, that must be the subject of another paper.

\section{References}

[1] Anderson E, Barbour J, Foster B Z and Ó Murchadha N 2002 "Scale-Invariant Gravity: Geometrodynamics", Class. Quantum Grav. 200320 1571, arXiv:gr-qc/0211022

[2] Barbour J, Foster B Z and Ó Murchadha N 2002 Class. Quantum Grav. 19 3217; "Relativity without relativity", arXiv:gr-qc/0012089

[3] Anderson E and Barbour J 2002 Class. Quantum Grav. 19 3249; "Interacting vector fields in relativity without relativity", arXiv:gr-qc/0201092

[4] Weyl H 1918 Sitzungsber. d. Preuss. Akad. d. Wissensch. 465 (There are English translations and commentaries of Weyl's papers togther with much interesting material on gauge theory in: O' Raifeartaigh L 1997 The Dawning of Gauge Theory (Princeton: Princeton University Press)

[5] Einstein's objection was reported by Weyl at the end of 4

[6] Dirac P A M 1973 Proc. R. Soc. A 333403

[7] Brans C and Dicke R 1962 Phys. Rev. 124935

[8] Wetterich C 1988 Nucl. Phys. B302 645 668; Bachmüller W and Dragon N 1989 Nucl. Phys. B321 207

[9] Weyl H 1919 Ann. d. Physik 59101

[10] Weyl H 1949 Philosophy of Mathematics and Natural Science (Princeton, Princeton University Press), p. 83

[11] Poincaré H 1905 Science and Hypothesis (London, translated from the French edition of 1902)

[12] Mach E 1883 Die Mechanik in ihrer Entwicklung historisch-kritsch dargestellt (Leipzig: Barth); 1893 The Science of Mechanics (Chicago: Open Court)

[13] Barbour J 2001 "On general covariance and best matching", in Physics Meets Philosophy at the Planck Length, eds. Callender C and Huggett N (Cambridge: Cambridge University Press)

[14] Einstein A 1955 The Meaning of Relativity (London: Methuen), Appendix II

[15] Schrödinger E 1925 Annalen der Physik 77325 (English translation in: Mach's Principle: From Newton's Bucket to Quantum Gravity, eds. Barbour J and Pfister H (Boston: Birkhäuser)

[16] Hughes V, Robinson H, and Beltran-Lopez V 1960 Phys. Rev. Lett. 4 342; Drever R 1961 Phil Mag. 6683

[17] Barbour J and Bertotti B 1982 Proc. R. Soc. A 382295

[18] Hertz H 1956 The Principles of Mechanics (New York: Dover) (German original 1895) 
[19] Lanczos C 1949 The Variational Principles of Mechanics (University of Toronto Press, Toronto)(also available from Dover, New York)

[20] Barrow J D 2002 The Constants of Nature (London: Jonathan Cape)

[21] Fulton T, Rohrlich F, and Witten L 1962 Reviews of Modern Physics 34442

[22] Chenciner A and Simó C 2001 Private communication

[23] Dziobek O 1888 Die mathematische Theorien der Planeten-Bewegungen (Leipzig: J A Barth); English translation: Mathematical Theories of Planetary Motions (New York: Dover).

[24] Barbour J 1994 Class. Quantum Grav. 112875

[25] Barbour J 1999 The End of Time (London: Weidenfeld and Nicolson; New York: Oxford University Press)

[26] Dirac P A M 1964 Lectures on Quantum Mechanics (New York: Yeshiva University)

[27] Poincaré H 1898 Rev. Métaphys. Morale 61 (English translation 1913: "The Measure of Time" in The Value of Science (New York: Science Press)

[28] Clemence G 1957 Rev. Mod. Phys. 292

[29] Littlejohn R and Reinsch M 1997 Rev. Mod. Phys. 69213

[30] Marchal C 1990 The Three-Body Problem (Amsterdam: Elsevier)

[31] Moeckel R 1988 Contemporary Mathematics 811

[32] Einstein A 1917 Sitzungsber. d. Preuss. Akad. d. Wissensch. 1142

[33] Chenciner A 1998 Reg. Chaotic Dynamics 393

[34] Barbour J and Bertotti B 1977 Nuovo Cimento 38B 1

[35] Planck M 1899 Sitzungsber. d. Preuss. Akad. d. Wissensch. 5 440; Ann. d. Physik 1169

[36] Rees M J and Sciama D W 1968 Nature 217 511; Padmanabhan T 1993 "Structure Formation in the Universe" (Cambridge: Cambridge University Press).

[37] Kendall D 1984 Bull. London Math. Soc. 1681

[38] Tanner G, Richter K and Rost J-M 2000 Rev. Mod. Phys. 72497

[39] DeWitt B 1967 Phys. Rev. 1601113

[40] Richard Montgomery 1999 Private communication. 